Elsevier required licence: (c) 2019

This manuscript version is made available under the CC-BY-NC-ND 4.0 license

http://creativecommons.org/licenses/by-nc-nd/4.0/

The definitive publisher version is available online at

https://doi.org/10.1016/j.energy.2019.02.080 


\title{
Modeling for chaotic time series based on linear and nonlinear framework: Application to wind speed forecasting
}

\author{
Ping Jianga ${ }^{\mathrm{a}}$, Biao Wang ${ }^{\mathrm{a},{ }^{*}}$ Hongmin $\mathrm{Li}^{\mathrm{a}}$, Haiyan $\mathrm{Lu}^{\mathrm{b}}$ \\ ${ }^{a}$ School of Statistics, Dongbei University of Finance and Economics, Dalian, China \\ b School of Software, Faculty of Engineering and Information Technology, University of Technology, \\ Sydney, Australia \\ * Corresponding author. Address: School of Statistics, Dongbei University of Finance and Economics, \\ Dalian 116025, China \\ Tel.: +86 15382250021. \\ E-mail address: wangbiaofugg@163.com.
}

\begin{abstract}
:
Wind-speed forecasting plays a crucial part in improving the operational efficiency of wind power generation. However, accurate forecasts are difficult owing to the uncertainty of the wind speed. Although numerous investigations of wind-speed forecasting have been performed, many of the previous studies used wind-speed data directly to make forecasts, which were rarely based on the structural characteristics of the data. Therefore, in this study, a hybrid linear-nonlinear modeling method based on the chaos theory was successfully employed to capture the linear and nonlinear factors hidden in chaotic time series. Before the forecast, the noise in the data was removed using a decomposition algorithm. Then, through the phase-space reconstruction, the one-dimensional time series were extended to the multi-dimensional space to determine the utilization form of the data. Finally, Holt's exponential smoothing based on the firefly optimization algorithm and support vector regression were combined to predict the wind speed. The experimental results show that the proposed model is not only better than the comparison models but also has great application potential in the wind power generation system.
\end{abstract}

Keywords: Wind speed; Data characteristics; Linear and nonlinear; Chaotic time series; Phase space reconstruction 


\section{Introduction}

With the rapid increase in the demand for energy, the potential of wind power is gaining increased attention. The use of wind energy to generate electricity can reduce the consumption of traditional energy resources as well as environmental pollution. However, the effective use of wind energy is not simple. Owing to its inherent discontinuity and limited predictability, the integration of the electric system poses a profound challenge to operations and planning practices [1]. The power system will benefit from efficient and accurate wind-speed forecasting for mitigating unstable operation, especially when the generated electricity is incorporated into the grid system [2]. Additionally, a correlation study showed that if the wind-speed prediction accuracy is improved by $10 \%$, the expected value of wind power generation can be increased by approximately $30 \%$ [3]. Thus, wind-speed forecasting has become a popular research topic owing to its importance for the efficiency of wind power generation [4].

In view of the significant demand, numerous researchers have studied wind-speed predictions both theoretically and practically. Wind-speed forecasting models are diverse and can be classified into three major categories [5]: physical approaches, statistical methods, and artificial intelligence methods. Physical models based on numerical weather prediction (NWP) are often used for long-term wind-speed forecasting [6]. However, the complexity of the calculation process and high costs limit the application of NWP models. In contrast, the statistical model is based on the sample data and involves obtaining the functional relationship among the variables, usually via a conventional time series analysis, e.g., exponential smoothing (ES) [7], autoregressive moving average [8], and autoregressive integrated moving average (ARIMA) [9]. These are all inferred from the historical data to determine the trend of the wind speed. However, the factors affecting the wind-speed series are diverse and require complex functions to capture the functional relationships between variables [10]. Fortunately, with the rapid development of computer technology, various artificial intelligence prediction methods have been applied to wind-speed forecasting, such as the artificial neural network (ANN) [10-12], extreme learning machine (ELM) [13], and support vector machine (SVM) [14]. They can capture hidden nonlinear relationships in data through machine learning and pattern recognition [15]. However, each method has insurmountable disadvantages owing to its inherent properties, and it is difficult for a single prediction model to completely capture hidden information in a time series [16].

Statistical methods based on assumptions tend to exhibit poor prediction performance for predicting highly nonlinear time series. To highlight the advantages of the model and compensate for its shortcomings, it is effective to establish a model structure combining linearity and nonlinearity. In 2003, Zhang et al. [17] proposed a model combining the ARIMA and an ANN to capture linear and nonlinear factors in a time series. Since then, the combination of linear and nonlinear models has been widely used for wind-speed forecasting. In addition, various optimization algorithms are used to optimize the initial weights and thresholds of the ANN, which improves the prediction performance of the model [18]. In 2017, Zhang et al. [19] applied an ELM based on a backtracking search optimization algorithm to wind-speed forecasting and experimentally demonstrated its good prediction performance.

Furthermore, most of the previous studies focused only on improving the accuracy of the model, ignoring the characteristics of the wind-speed time series. Thus, to discover the useful information in the data, it is necessary to preprocess and characterize the data before prediction [20]. Therefore, data denoising methods such as empirical mode decomposition (EMD) aim to reduce random disturbances in the data sequence 
and increase the prediction accuracy, and are considered to be applicable to wind-speed forecasting [21]. Compensating for the deficiencies of EMD, ensemble EMD (EEMD) is a noise-assisted data analysis method that has been widely applied to filter out the disturbing factors of wind-speed series [22, 23]. For instance, Wang et al. [24] utilized an EEMD method in which the raw data are decomposed into signals with different frequencies. Although EEMD has been greatly improved and widely applied in many areas, it has the drawback that noise cannot be effectively removed. Thereby, the complete EEMD with adaptive noise (CEEMDAN) was proposed by Torres et al. [25]. Zhang et al. [26] proposed a combined forecasting model. CEEMDAN is employed to divide the original wind-speed data into finite subsequences, and then the fullparameter fraction forecasting model is applied to forecast each decomposition. Ye et al. [27] compared the four decomposition methods (EMD, EEMD, CEEMD, and CEEMDAN) and combined the models to predict the wind speed. The results showed that CEEMDAN-support vector regression (SVR) had good prediction performance and a high operational efficiency.

The foregoing review of the literature indicates the following. (1) Physical forecasting methods need to collect a large amount of data, mainly applied to regional prediction. Thus, they do not have high economic value for wind power companies. (2) The traditional statistical models do not uncover information hidden in data with noise components and cannot effectively improve the forecasting accuracy. (3) ANNs have difficulty determining a suitable network structure, and may easily enter an overfitting state. (4) The artificial intelligence model improves the reliability of the neural network structure by combining optimization algorithms but ignores the importance of the data preprocessing stage.

In light of the aforementioned limitations, this study proposes a novel hybrid model (CEE-CC-FHS) for wind-speed forecasting. First, the chaotic characteristics of windspeed time series are determined by calculating the maximum Lyapunov exponent (MLYE) [28]. Numerical simulation results show that the time series can be used as a chaotic system for predictive analysis [29,30]. The raw wind-speed sequence is decomposed into a finite component set via the CEEMDAN method to eliminate the interference factor, and then the information contained in the sequence is mined by determining the extended form of the sequence using the $\mathrm{C}-\mathrm{C}$ method. On this basis, a linear and nonlinear modeling framework and a hybrid model based on firefly optimization were proposed. The simulation results were compared with those for other methods. The experimental results indicate that the hybrid model can has excellent prediction performance.

\section{The novelty and originality of the proposed method are explained as follows.}

- A novel combined model based on linear and nonlinear frameworks is constructed without considering the statistical distribution. The experimental simulation results confirm that the proposed model has excellent prediction accuracy.

- An outstanding model input structure can be obtained. Through phase-space theory, the single-dimensional wind-speed time sequences are converted into a matrix time series to improve the generalization ability. The matrix time series can more clearly describe the sequence characteristics, increasing the accuracy of the forecasting results.

- The hybrid "linear and nonlinear" modeling framework based on the firefly optimization algorithm can capture the linear and nonlinear modes contained in the time series well.

- The practicability and effectiveness of the proposed forecasting model were confirmed. A feasible method for accurate wind-speed prediction is provided. 
The structure of this paper is as follows. The theory of the combined forecasting models for wind-speed forecasting is introduced in Section 2. The proposed combined forecasting model is described in Section 3. Detailed experimental analysis is presented in Section 4. Analysis and discussions are presented in Section 5. Conclusions are presented in the last section.

\section{Methodology}

In this section, the methods and principles of CEEMDAN, PSR, the firefly algorithm (FA), Holt, and SVR are detailed.

\subsection{CEEMDAN}

Huang et al. [31] proposed the application of EMD for analyzing nonlinear data. As an improved method for EMD, EEMD was first proposed in [32]. EEMD divides the sequence into different components when the white noise is uniformly distributed throughout the time-frequency space. The details of EEMD are presented in [33].

Different distributions of white Gaussian noise with zero mean and unit variance are represented as $w^{i}(n), i=1,2, \ldots, I$. The standard deviation of the white Gaussian noise is $\varepsilon_{0}$. The decomposition steps of CEEMDAN are as follows [34].

Step 1: The above $I$ signals are decomposed via EMD to obtain their first mode and calculate $x(n)+\varepsilon_{0} w^{i}(n)$. The formula is as follows:

$$
\operatorname{IMF}_{1}(n)=\frac{1}{I} \sum_{i=1}^{I} I M F_{1}^{i}(n)=\overline{I M F_{1}}(n) .
$$

Step 2: The first residue can be obtained:

$$
r_{1}(n)=x(n)-\mathcal{I M F}_{1}(n) .
$$

Step 3: Decompose the realizations $r_{1}(n)+\varepsilon_{1} E_{1}\left(w^{i}(n)\right), i=1,2, \mathrm{~K}, I$ up to their first EMD mode. $\varepsilon_{1}$ is the standard deviation of white Gaussian noise at the first stage. $\mathrm{MMF}_{2}(n)$ is expressed as follows:

$$
\text { IMF } F_{2}(n)=\frac{1}{I} \sum_{i=1}^{I} E_{1}\left(r_{1}(n)+\varepsilon_{0} E_{1}\left(w^{i}(n)\right)\right) .
$$

Step 4: With $K$ being the total number of modes, calculate the $k^{\text {th }}$ residue as follows:

$$
r_{k}(n)=r_{k-1}(n)-\mathcal{I} M F_{k}(n), k=2,3, \mathrm{~K}, K .
$$

Step 5: Decompose the realizations $r_{k}(n)+\varepsilon_{k} E_{k}\left(w^{i}(n)\right)$ with $i=1,2, \ldots, I$, up to their first EMD mode. The $(k+1)^{\text {th }}$ mode can be calculated as follows:

$$
\text { I } M F_{k+1}(n)=\frac{1}{I} \sum_{i=1}^{I} E_{1}\left(r_{k}(n)+\varepsilon_{k} E_{k}\left(w^{i}(n)\right)\right) .
$$

Step 6: Repeat steps 4 to 6 until the residue becomes a monotonic function, so that it is impossible to further extract the $I M F$. Assuming that $k$ and $r_{k}(n)$ represent the total number of modes and the final residue, respectively, the input $x(n)$ is expressed as

$$
x(n)=\sum_{k=1}^{K} \mathcal{I} M F_{k}(n)+r_{k}(n) .
$$

Eq. (6) completes the decomposition of the original data.

\subsection{Phase-space reconstruction}

The phase-space reconstruction (PSR) theory is considered to be a useful method 
for reconstructing chaotic time series [35].

Definition 1. The given time series $x=\left\{\mathrm{x}_{1}, \mathrm{x}_{2}, \ldots, \mathrm{x}_{N}\right\}$ can be extended to a matrix time series as follows:

$$
X=\left[\begin{array}{cccc}
x_{1} & x_{2} & \mathrm{~L} & x_{N-(m-1) \tau} \\
x_{1+\tau} & x_{2+\tau} & \mathrm{L} & x_{N-(m-2) \tau} \\
\mathrm{M} & \mathrm{M} & \mathrm{M} & \mathrm{M} \\
x_{1+(m-1) \tau} & x_{2+(m-1) \tau} & \mathrm{L} & x_{N}
\end{array}\right],
$$

where $M=N-(\mathrm{m}-1) \tau$. The parameter $m$ represents the embedding dimension, and $\tau$ is the delay time.

In the study, the $\mathrm{C}-\mathrm{C}$ method [36] is employed to reconstruct the phase space by applying correlation integrals to estimate $m$ and $\tau$ simultaneously.

Definition 2. The correlation integral for the embedded time series is as follows:

$$
C(m, N, r, t)=\frac{2}{M^{\prime}\left(M^{\prime}-1\right)} \sum_{1 \leq i \leq j \leq M^{\prime}} \theta\left(r-d_{i j}\right), r>0,
$$

where $d_{i j}=\left\|X_{i}-X_{J}\right\|$ represents the sup-norm, $\tau$ is the index lag, and $\theta(x)=\left\{\begin{array}{l}0, x<0 \\ 1, x \geq 0\end{array}\right.$. The correlation integral is a cumulative distribution function, and the Euclidean distance between any two points in the phase space is less than $r$.

Definition 3. The given sequence $x$ is divided into $t$ disjoint subsequences to form the matrix time series. Let us structure a statistic $S(m, N, r, t)$, which can be calculated via the following formula. The correlation integral $S(m, N, r, t)$ can be expressed as follows:

$$
S(m, N, r, t)=\frac{1}{t} \sum_{s=1}^{t}\left[C_{s}\left(m, \frac{N}{t}, r, t\right)-C_{s}^{m}\left(1, \frac{N}{t}, r, t\right)\right] .
$$

Select two radii $r$ corresponding to the maximum and minimum values, and define the difference as follows:

$$
\Delta S(m, t)=\max \left\{S\left(m, r_{j}, t\right)\right\}-\min \left\{S\left(m, r_{j}, t\right)\right\},
$$

where $\Delta S(m, t)$ indicates the maximum deviation of the radius $r$.

\subsection{Firefly algorithm}

The FA was proposed by Yang as a heuristic algorithm to imitate firefly luminescence behavior in nature and structure [37]. The FA can be used to solve nonlinear optimization problems under various constraints. The algorithm is a special particle swarm optimization (PSO) algorithm that is easy to understand and implement. Fireflies are described by the following three idealization rules.

1. All fireflies are unisexual and can be attracted to each other.

2. The attraction ability of fireflies depends on brightness, i.e., fireflies move in the direction of bright fireflies. At the same time, attractiveness is inversely proportional to distance.

3. The brightness of fireflies is determined by the fitness function value.

Definition 1. The function of the luminance of the firefly is expressed as follows:

$$
I=I_{0} e^{-\gamma r_{i j}^{2}},
$$

where $I(r)$ is the brightness of the firefly; $r$ the distance between two fireflies $i$ 
and $j ; I_{\mathrm{o}}$ is the nature brightness; and $\gamma$ is the attractive coefficient of brightness.

Definition 2. The attractiveness of the firefly is as follows:

$$
\beta=\beta_{0} e^{-\gamma r_{i j}^{2}} .
$$

The distance between any two fireflies $i$ and $j$ is expressed by the Descartes function. The formula is as follows:

$$
r_{i j}=\left\|x_{i}-x_{j}\right\|=\sqrt{\sum_{k=1}^{d}\left(x_{i, k}-x_{j, k}\right)^{2}} .
$$

Hence, $x_{i, k}$ defines the $k^{\text {th }}$ component of the spatial coordinate $x_{i}$ of the $i^{\text {th }}$ firefly.

Definition 3. The trajectory of firefly $i$ attracted by firefly $j$ is expressed as follows:

$$
x_{i}=x_{i}+\beta \times e^{-\gamma r_{i j}^{2}} \times\left(x_{j}-x_{i}\right)+\alpha \times(\text { rand }-1 / 2),
$$

where $\alpha$ is a random parameter.

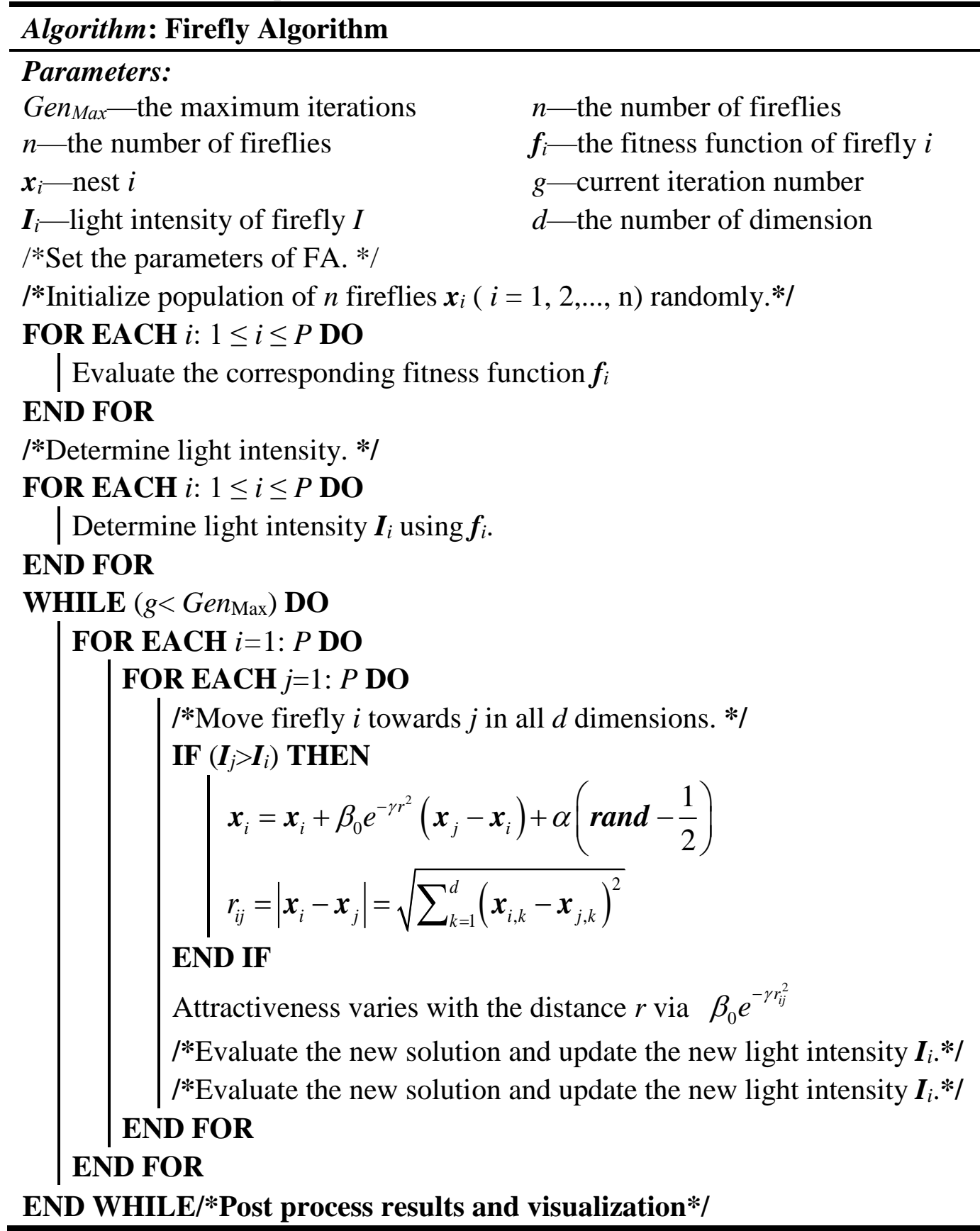




\subsection{Holt's ES method}

The ES model is widely used in business, environmental science, and other fields because of its simple structure and effectiveness. Holt's ES model was proposed in 1957 [38].

Unlike the general ES model, Holt's ES model involves direct smoothing of the trend data and a prediction of the original time series. It assumes that all the known data influence the prediction value. The short-term data have great influence on the prediction value, and the long-term data have little influence on the prediction value.

Definition 1. The specific formulas are as follows:

$$
\begin{gathered}
S_{t}=\alpha X_{t}+(1-\alpha)\left(S_{t-1}+T_{t-1}\right) \\
T_{t}=\gamma\left(S_{t}-S_{t-1}\right)+(1-\gamma) T_{t-1},
\end{gathered}
$$

where $0<\alpha$ and $\gamma<1$ are the smoothing parameters. In this model, $\alpha$ determines the average length of the estimate level, and $\gamma$ determines the smoothing trend.

Definition 2. A straightforward approach for finding the optimal values of both $\alpha$ and $\beta$ constrained to the range $(0,1)$ is to search for the parameter combination that minimizes the sum of squared errors of predictions:

$$
\operatorname{error}(\alpha, \gamma)=\sum_{i=1}^{n}\left(X_{i}^{\prime}-X_{i}\right)^{2}
$$

where $X_{t+1}=S_{2}+T_{2}$. The starting values for $S_{2}$ and $T_{2}$ are typically taken as $X_{2}$ and 0 , respectively.

\subsection{Support vector regression}

Vapnik [39] proposed SVR, which is derived from SVMs. The advantage of SVR is its ability to model nonlinear relations. SVR is based on the principle of structural risk minimization, and the regression task of support vector classification is completed by introducing the $\varepsilon$ insensitive loss function. The following is a brief description of SVR.

Definition 1. The decision function of support vector regression is

$$
f(x)=\langle w, \varphi(x)\rangle+b .
$$

Here, $\varphi(x)$ represents the nonlinear mapping of the input space to the highdimensional space, $w$ represents the weight, and $b$ is the offset.

Definition 2. To estimate $w$ and $b$, the following formula is minimized:

$$
R(f)=\frac{1}{2}\|w\|^{2}+C \frac{1}{n} \sum_{i=1}^{n} L_{\varepsilon}\left(y_{i}, f\left(x_{i}\right)\right),
$$

where $L_{\varepsilon}$ is a loss function, and $C$ is a penalty factor. A greater $C$ value indicates a greater penalty for data that exceed $L_{\varepsilon}$. The $\varepsilon$ insensitive loss function is used as the problem of structural minimum risk estimation.

Definition 3. The Lagrange equation is introduced, the partial derivative of the relevant parameters is obtained, and the regression estimation function of the SVM is obtained:

$$
f(x)=\sum_{i=1}^{N}\left(\overline{\alpha_{i}}-\overline{\alpha_{i}^{*}}\right) K\left(x_{i}, x\right)+b
$$

where $\overline{\alpha_{i}}, \overline{\alpha_{i}^{*}} \geq 0(i=1,2, \cdots, N)$ is a Lagrange multiplier, $x$ is a kernel function, 
and $N$ is the number of input parameters. Different types of kernel functions can be selected to obtain different nonlinear decision learning machines. Because the radial basis function has good adaptability and a good convergence area in low- and highdimensional spaces, the Gauss radial basis function is used in this study.

\section{Framework of proposed model}

The proposed novel model framework aims to improve the forecasting accuracy and the utilization of wind energy. The model considers the chaotic characteristics in the time series. First, the original data are preprocessed, and then the data input form that can provide more real information is determined using PSR technology. Finally, the "linear and nonlinear" framework is constructed for prediction based on the optimization algorithm.

Owing to the irregularity and randomness in the original observation data before model forecasting, the Lyapunov index is employed to quantitatively describe the chaotic phenomenon of wind-speed series, which is an important quantitative measure of system dynamics. To eliminate noise to the greatest extent possible, a noise-assisted data analysis method with CEEMDAN is developed to remove noise and extract valuable information in the first phase of forecasting. After CEEMDAN, a smooth series is obtained by decomposing the original wind speed and removing the highfrequency $I M F$. Furthermore, the rest of the $I M F$ s are refactored to obtain preprocessed data. The process is shown in Fig. 1. PSR technology is used to analyze the collected datasets, and the sequence can be regarded as a chaotic system. Therefore, the C-C method determines the optimal input form of the predicted structure of the design, which is a technique for reconstructing the phase space. Through the obtained optimal embedding dimension $(m)$ and optimal delay time $(\tau)$, the sequence features can be described more clearly. A schematic of the input structure of PSR is shown in Fig. 1.

The linear and nonlinear forecasting models are combined to obtain an outstanding tool for capturing information hidden in the data and improving the predictive performance [40]. It is advisable to regard the time series $X_{t}(t=1, \ldots, n)$ as consisting of two parts: a linear component and a nonlinear component. That is,

$$
X_{t}=L_{t}+N_{t},
$$

where $L_{t}$ denotes the linear components, and $N_{t}$ denotes the nonlinear components.

Considering the advantages of Holt's ES method for capturing the linear and nonlinear factors of the SVR model, the proposed hybrid model employs the firefly optimization algorithm. The steps of forecasting in the proposed CEE-CC-FHS method are shown in Fig. 1 and described as follows.

Step 1: The linear component of forecasting $L_{t}$ is determined using Holt's method. The residuals are as follows:

$$
e_{t}=X_{t}-\dot{L}_{t} \text {. }
$$

The residuals $e_{t}$ contain information, excluding the linear factor. $\widehat{L}_{t}$ represents the prediction results of Holt's method.

Step 2: SVR is used to determine the nonlinear component $e_{t}$.

$$
e_{t}=f\left(e_{t-1}, \mathrm{~K}, e_{t-d}\right)+\varepsilon_{t}
$$

Here, $f(\square)$ represents the nonlinear process, and $\varepsilon_{t}$ represents a stochastic error. We input $e_{t-1}, \ldots, e_{t-d}$ into the SVR model to obtain the output result $e_{t}$, as described in Section 2.5. The residual predicted by the SVR model contains the nonlinear component. 
Step 3: Combining prediction results of linear and nonlinear models: $\widehat{e}_{t}$ represents the predicted residuals. Therefore, the results predicted by the proposed CEE-CC-FHS model are given by

$$
\dot{X}_{t}=\dot{L}_{t}+e_{t} .
$$
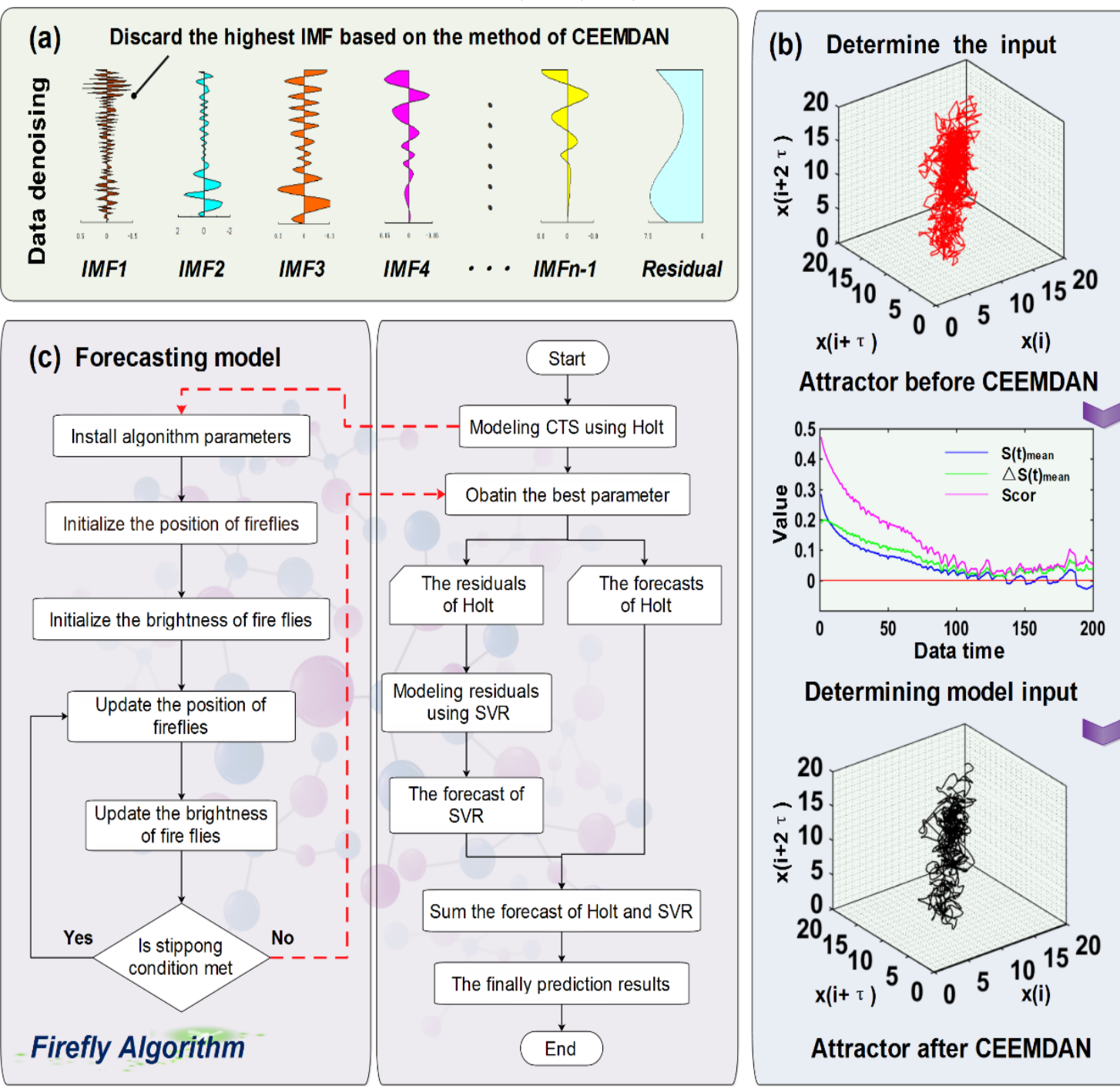

Determining model input

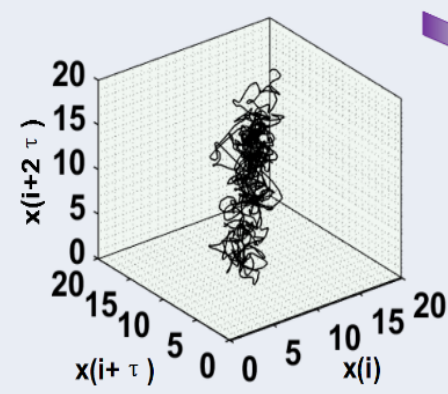

Attractor after CEEMDAN

Fig. 1. Flow chart of the proposed combined model.

\section{Experimentation and analysis}

The experimental analysis is detailed in this section. In Section 4.1, a basic statistical description of the datasets is provided. The statistical criterion of the prediction level is described in Section 4.2. The implementations of numerical modeling for the prediction of wind speed are described in Sections 4.3-4.5. All the numerical simulations were implemented using MATLAB R2017a software.

\subsection{Data description}

Experimental datasets collected from wind farms in China are used as samples to evaluate the effectiveness of the proposed model. The trends of the wind-speed series for three locations are illustrated in Fig. 2. The descriptive statistics (mean, minimum, maximum, standard deviation, MLYE, and complexity) for the three sites are presented in Table 1. The values of the MLYE, which describe the chaotic characteristics of the time series, are all positive in the experimental datasets. 
Table 1

Descriptive statistical of the data set.

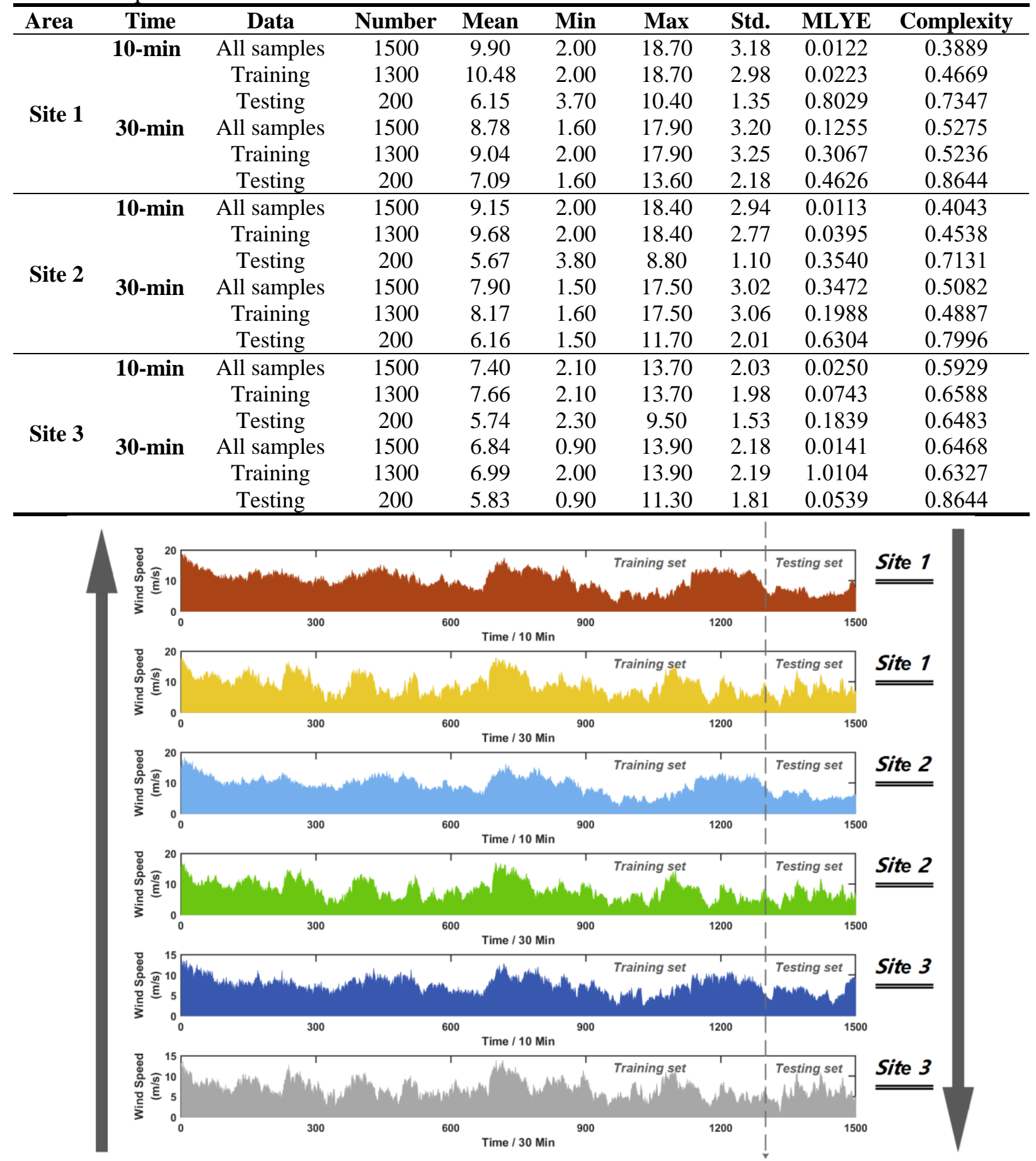

Fig. 2. Test Site: wind speed data.

Table 1 indicates basic characteristics of the experimental datasets. However, it is insufficient to survey the wind-speed patterns in Table 1. Therefore, the frequency distribution of the wind-speed series and the corresponding fit for the distributions (Weibull, gamma, normal) are shown in Fig. 3. In all cases, the change of the data collected from different sites or wind-speed patterns at the same location can be displayed by shape parameters and scale parameters, as well as normal distribution parameters. The results of Table 1 and the distribution parameters of Fig. 3 indicate that the differences between different wind-speed time series are significant. Therefore, it 
can be concluded that the three test points of the comparison are meaningful, not invalid.
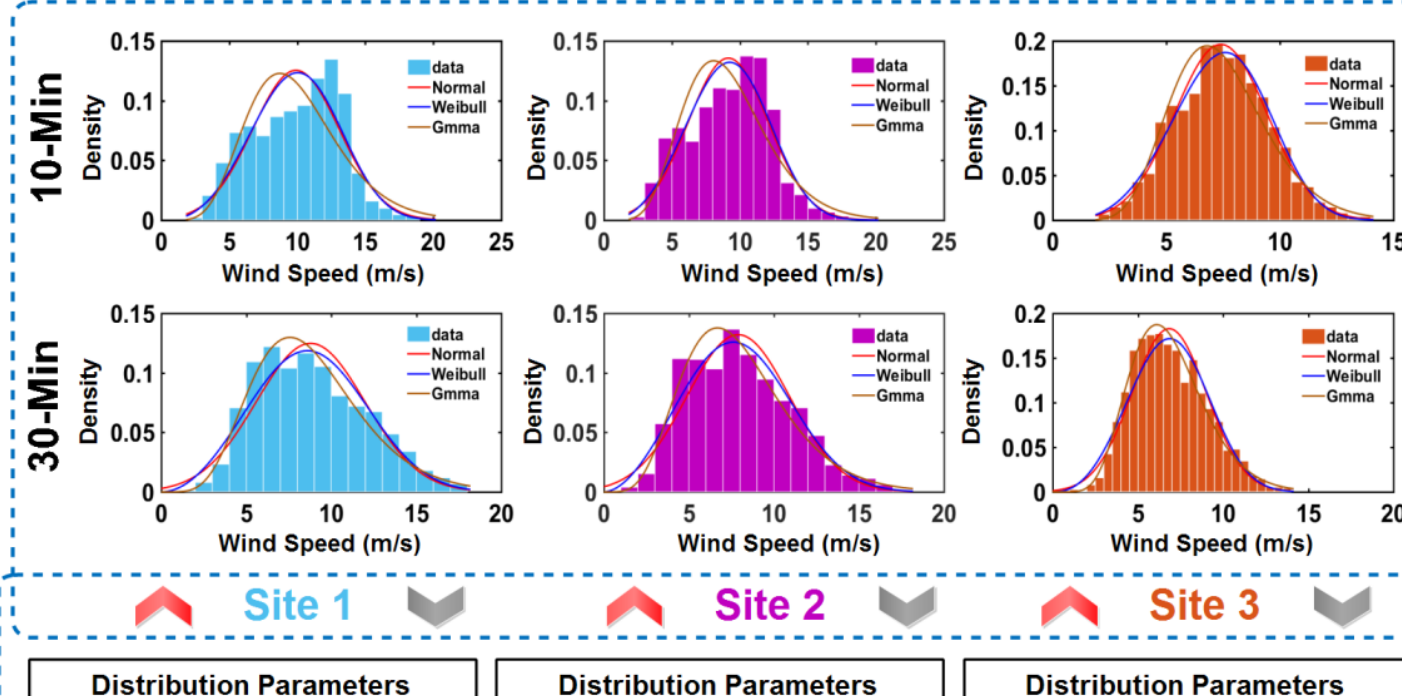

Site 2

Site 3
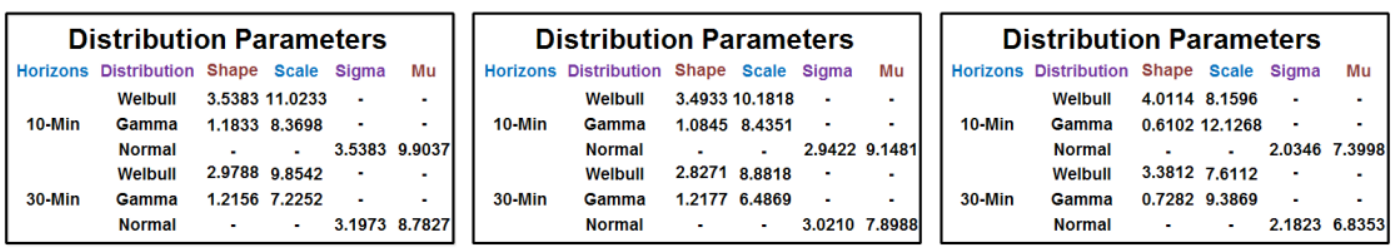

Fig. 3. Distribution fitting of wind speed series.

\subsection{The evaluation criteria}

The evaluation criterion is used to intuitively measure the accuracy of model forecasting. Although a multitude of evaluation criteria have been applied, no single evaluation criterion has proven to be effective for all situations. Therefore, in this study, three common error indices - the mean absolute error (MAE), mean square error (MSE), and mean absolute percentage error (MAPE) - are applied to evaluate the forecasting performance of the proposed model. The evaluation criteria are given as follows:

$$
\begin{gathered}
M A E=\frac{1}{N} \sum_{\mathrm{i}=1}^{N}\left|\hat{y}_{i}-y_{i}\right| \\
M S E=\frac{1}{N} \sum_{i=1}^{N}\left(\hat{y}_{i}-y_{i}\right)^{2} \\
M A P E=\frac{1}{N} \sum_{i=1}^{N}\left|\frac{\hat{y}_{i}-y_{i}}{y_{i}}\right| \times 100 \%,
\end{gathered}
$$

where $N$ represents the length of the forecasting series; $\hat{y}_{i}(i=1,2, \ldots, N)$ represents the forecast values, and $y_{i}(i=1,2, \ldots, N)$ represents the observed wind speed during the same period.

The number of predictions within a certain error range is analyzed for comprehensive comparison. Additionally, the maximum permissible error (MPE) is introduced:

$$
M P E=\frac{y_{i}-\hat{y}_{i}}{y_{i}} \times 100 \%
$$

\subsection{Data feature processing}

As described in this subsection, the disturbance factors of the wind-speed series are removed via CEEMDAN, and then the optimal input of the model is determined by reconstructing the phase space. The process is as follows. 
Step 1: Data denoising. The noise component in the wind-speed series greatly affects the model prediction performance. The wind-speed series is treated as a signal and decomposed into ten $I M F_{\mathrm{s}}$ with different frequency ranges. The original windspeed time series is decomposed via CEEMDAN into several $I M F_{\mathrm{s}}$, such as $I M F_{1}, \ldots$, $I M F_{n-1}$ and $R_{e s}$. By eliminating the $M I F_{1}$ to smooth the original time series, the prediction efficiency and accuracy can be improved.

Step 2: Data expansion. The different input forms have a significant effect on the predictive performance of the model. Therefore, to fully reveal the information in the time series, the time series is expanded to a high-dimensional space via PSR technology. Thus, the optimal embedding dimension and time window can be determined through the C-C method. PSR is used to transform the chaotic wind-speed sequence into a matrix time series, and a wind-speed dynamic model with embedding dimension $m$, time window $\varpi$, and time delay $\tau$ is constructed. The maximum lag is set as 200 in the C-C method $S(t)_{\text {mean }}$, and $\Delta S(t)_{\text {mean }}$ and Scor are calculated to determine the embedding dimension $m$ and the time delay $\tau$.

Although there are many observable factors that influence changes in time series, the true regularity of the system can be extracted and recovered from the time series. A trajectory generated by a chaotic system will eventually undergo regular movement after a certain period of change, resulting in a regular, tangible trajectory (chaotic attractor). Therefore, the chaotic time series can reveal the evolution of hidden chaotic attractors through PSR. Furthermore, after the noise is removed via CEEMDAN, the attractor is sufficiently recovered to reveal the true trajectory of the sequence motion, as shown in Fig. 4. 


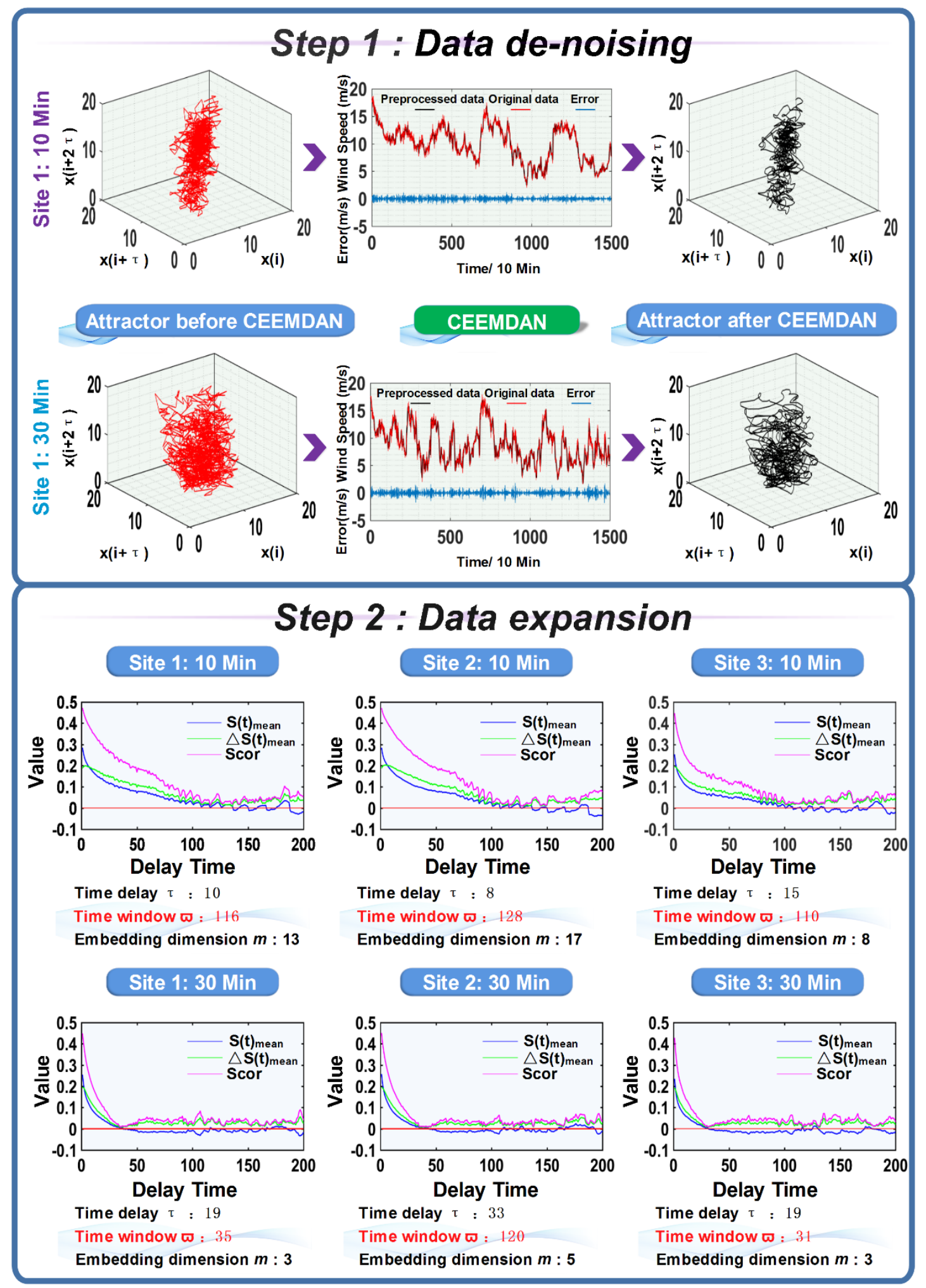

Fig. 4. Processing results of data features

\subsection{Experiment I}

The prediction module of the designed framework is predicted by combining linear and nonlinear models. To effectively verify the performance of the prediction module, four individual methods-ARIMA, Holt, the general regression neural network (GRNN), and SVR - and two hybrid methods-ARIMA-SVR and Holt-GRNN-are used as benchmark models for comparison. The aforementioned models are all 
processed using the same data preprocessing method before forecasting, which not only uses CEEMDAN denoising but also obtains the optimal input mode through the C-C method. Further comparisons are made, as follows.

a) From Table 2, the statistical error measures for six comparison models and the proposed models are reported. The MAPE values of the proposed model are smaller than those of the comparative models. For site 1, the MAPE of the CEE-CC-Holt model is $6.8999 \%$, while the MAPE of the CEE-CC-ARIMA model is $7.4737 \%$, indicating a $0.5738 \%$ improvement, as shown in Table 2 . For the hybrid CEE-CCARIMA-SVR and CEE-CC-FHG models, the MAPE values are $4.0905 \%$ and $3.5301 \%$, respectively, and produce increases of $1.7675 \%$ and $1.2071 \%$ compared with the proposed model.

b) For the MPE, a positive value indicates that the predicted value is lower than the actual value, and a negative value indicates that the predicted value is higher than the actual value. The results for the MPE within the error ranges of $\pm 2.5 \%, \pm 5 \%$, $\pm 7.5 \%$, and $\pm 10 \%$ for the three study sites are presented in Table 2 . For site $1,75.76 \%$ and $91.41 \%$ of the errors are within $\pm 5 \%$ between the measured values and the values predicted by the CEE-CC-FHG and the proposed models, respectively. The proposed model has more MPE values in the range of $\pm 10 \%$ than those of the compared models. The MPE values of CEE-CC-ARIMA, CEE-CC-Holt, CEE-CCGRNN, CEE-CC-SVR, CEE-CC-ARIMA-SVR, and CEE-CC-FHG are 70.00\%, $76.38 \%, 87.94 \%, 92.96 \%, 95.96 \%$, and $98.48 \%$, respectively.

c) Fig. 5 shows the error (MPE) and the residual distribution for a 10-min wind speed time series. For site 1, the MPE values of all seven models approximate to a normal distribution. However, the MPE distributions for the proposed model are more concentrated than those for the other models. For site 2, the MPE distribution of the proposed model more closely approximates the normal distribution compared with the other models.

d) The residual distribution in Fig. 5 shows that the points of the proposed model and CEE-CC-FHG are concentrated near the origin. Thus, a higher accuracy of fitting with the model indicates a higher accuracy of fitting with other models.

Remark. According to Table 2 and Fig. 5, the combination of linear and nonlinear models based on firefly optimization can improve the prediction accuracy. That is, the proposed model can accurately and stably forecast changes in future wind speeds. 

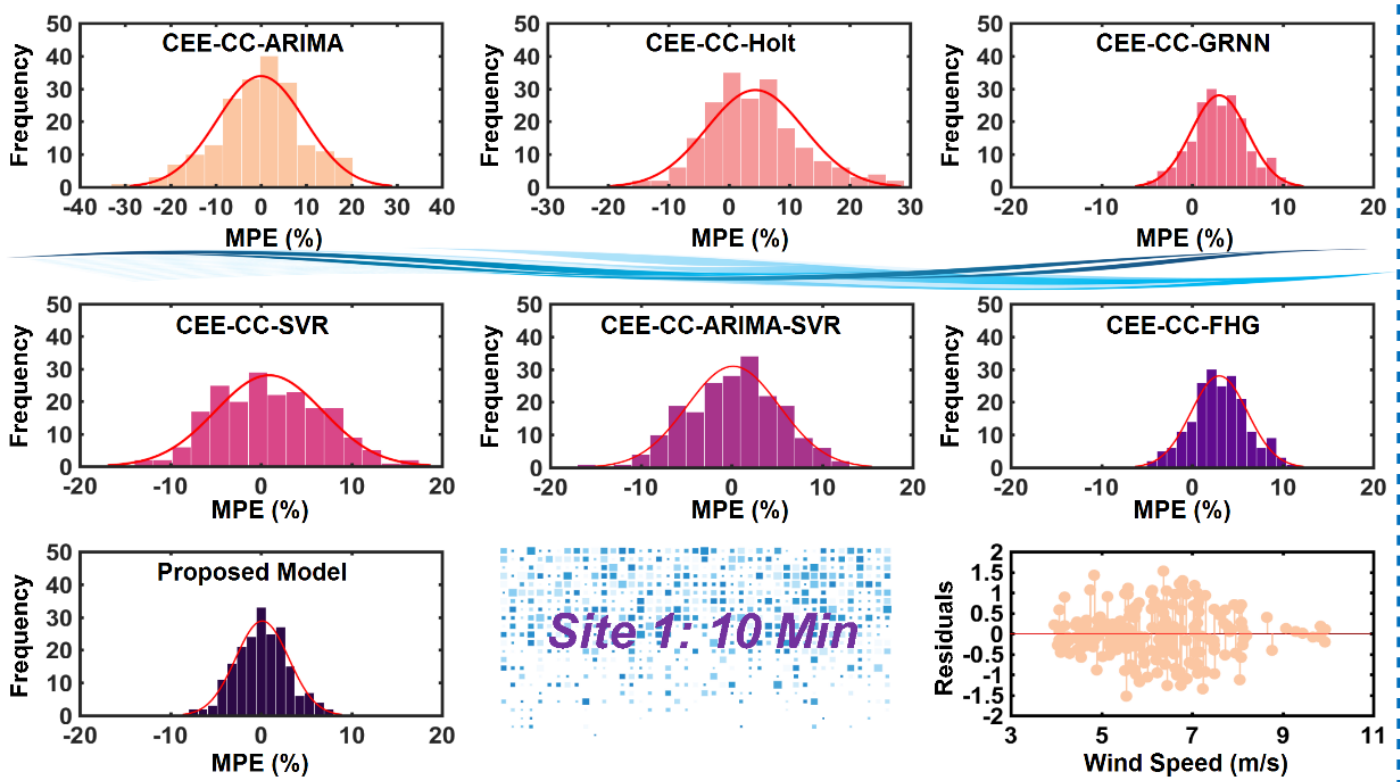

Experiment simulation
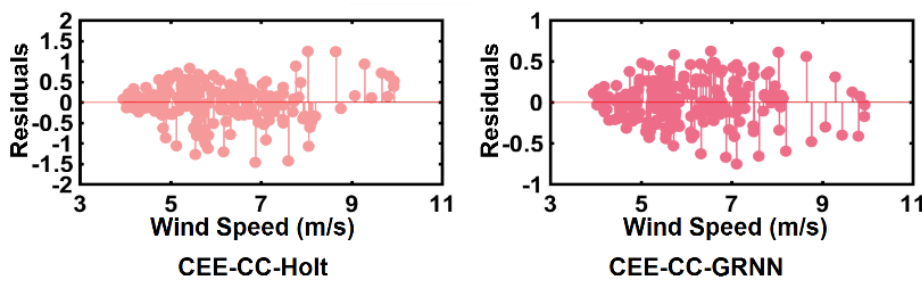

SSE: $43.2276 \quad$ Rsquare: 0.9083 CEE-CC-GRNN

SSE: 13.6670 Rsquare: 0.9598

Rmse: 0.2634 Adjrsquare: 0.9596

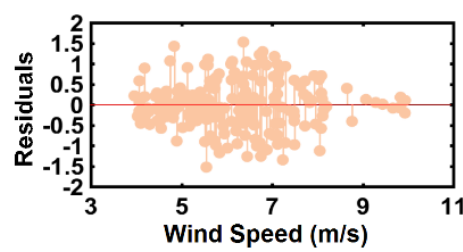

CEE-CC-ARIMA

SSE: $\mathbf{6 8 . 8 5 6 6}$ Rsquare: 0.8260

Rmse: 0.5897 Adjrsquare: 0.8251

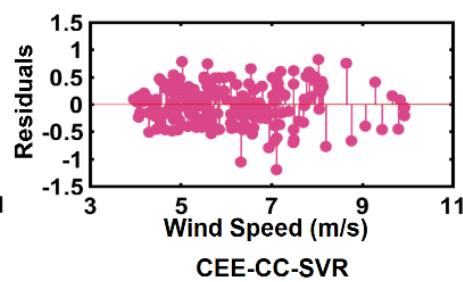

Rmse: 0.4684 Adjrsquare: 0.9078

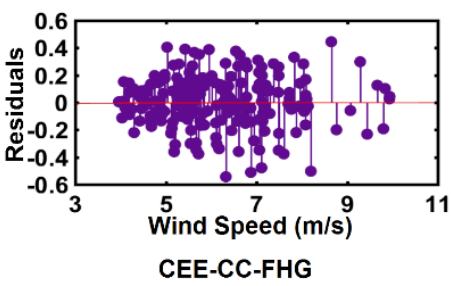

SSE: 25.1167 Rsquare: 0.9388

Rmse: 0.3571 Adjrsquare: 0.9385

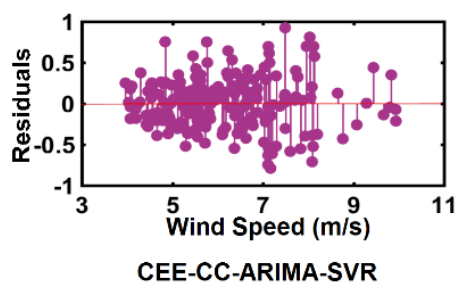

SSE: 19.5339 Rsquare: 0.9401

Rmse: 0.3157 Adjrsquare: 0.9398

SSE: 7.0261 Rsquare: 0.9799

Rmse: 0.1893 Adjrsquare: 0.9798

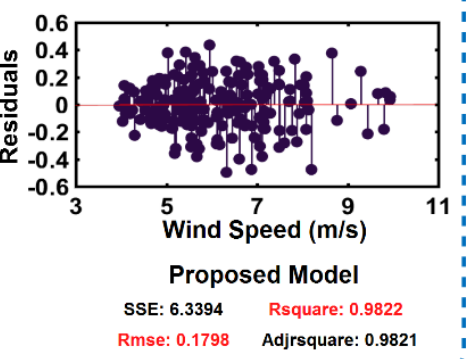

Fig. 5. The distribution of MPE and residual for site 
Table 2

Statistical error measures of the seven models for 10-Min wind speed.

\begin{tabular}{|c|c|c|c|c|c|c|c|c|}
\hline \multirow{2}{*}{ Area } & \multirow{2}{*}{ Model } & \multirow{2}{*}{$\begin{array}{l}\text { MAE } \\
(\mathbf{m} / \mathbf{s})\end{array}$} & \multirow{2}{*}{$\begin{array}{l}\text { MSE } \\
(\mathrm{m} / \mathbf{s})\end{array}$} & \multirow{2}{*}{$\begin{array}{c}\text { MAPE } \\
(\%)\end{array}$} & \multicolumn{4}{|c|}{ MPE (\% of errors in each margin) } \\
\hline & & & & & $\pm 2.5 \%$ & $\pm 5 \%$ & $\pm 7.5 \%$ & $\pm 10 \%$ \\
\hline \multirow{7}{*}{ Site1 } & CEE-CC-ARIMA & 0.4544 & 0.3457 & 7.4737 & 26.00 & 42.00 & 61.50 & 70.00 \\
\hline & CEE-CC-Holt & 0.4104 & 0.2929 & 6.8999 & 26.13 & 47.24 & 66.83 & 76.38 \\
\hline & CEE-CC-GRNN & 0.3261 & 0.1480 & 5.5156 & 24.62 & 49.25 & 70.85 & 87.94 \\
\hline & CEE-CC-SVR & 0.2955 & 0.1332 & 4.8748 & 27.64 & 56.28 & 78.89 & 92.96 \\
\hline & CEE-CC-ARIMA-SVR & 0.2540 & 0.1051 & 4.0905 & 38.89 & 64.14 & 86.87 & 95.96 \\
\hline & CEE-CC-FHG & 0.2113 & 0.0671 & 3.5301 & 40.40 & 75.76 & 91.92 & 98.48 \\
\hline & Proposed Model & 0.1413 & 0.0321 & 2.3230 & 62.12 & 91.41 & 99.49 & 100.00 \\
\hline \multirow{7}{*}{ Site2 } & CEE-CC-ARIMA & 0.6937 & 0.7903 & 12.3568 & 14.50 & 25.50 & 38.00 & 47.50 \\
\hline & CEE-CC-Holt & 0.3906 & 0.2417 & 7.3074 & 22.11 & 39.70 & 63.82 & 76.38 \\
\hline & CEE-CC-GRNN & 0.2526 & 0.0932 & 4.4897 & 31.66 & 61.81 & 83.92 & 93.97 \\
\hline & CEE-CC-SVR & 0.1717 & 0.0483 & 3.0571 & 51.26 & 79.90 & 94.47 & 98.99 \\
\hline & CEE-CC-ARIMA-SVR & 0.1490 & 0.0353 & 2.7020 & 54.55 & 85.86 & 97.47 & 99.49 \\
\hline & CEE-CC-FHG & 0.1619 & 0.0398 & 2.9644 & 45.96 & 82.32 & 96.46 & 99.49 \\
\hline & Proposed Model & 0.1207 & 0.0243 & 2.1587 & 65.66 & 91.41 & 98.48 & 100.00 \\
\hline \multirow{7}{*}{ Site3 } & CEE-CC-ARIMA & 0.5948 & 0.6106 & 10.7482 & 16.50 & 35.00 & 46.50 & 55.00 \\
\hline & CEE-CC-Holt & 0.3777 & 0.2343 & 7.1790 & 25.63 & 46.73 & 64.32 & 77.89 \\
\hline & CEE-CC-GRNN & 0.2094 & 0.0709 & 4.0289 & 43.72 & 68.84 & 83.92 & 92.46 \\
\hline & CEE-CC-SVR & 0.188 & 0.0614 & 3.5643 & 46.23 & 76.88 & 87.94 & 93.97 \\
\hline & CEE-CC-ARIMA-SVR & 0.1598 & 0.0401 & 2.9006 & 51.01 & 85.86 & 94.44 & 100.00 \\
\hline & CEE-CC-FHG & 0.1653 & 0.0398 & 3.0749 & 47.98 & 81.82 & 95.45 & 98.99 \\
\hline & Proposed Model & 0.1252 & 0.0249 & 2.2998 & 62.63 & 91.41 & 97.98 & 100.00 \\
\hline
\end{tabular}




\subsection{Experiment II}

In this subsection, to further illustrate the generalization ability of the proposed model, different data preprocessing techniques and optimization methods are compared. The importance of data preprocessing, the optimal input structure of the model, and the parameter optimization for prediction accuracy were verified by Experiment II. Five comparison models were used to validate the superiority of model structure. PSO (CEECC-PHS) was used to optimize the model parameters, compared with the optimization of the FA. The forecasting results for different models are presented in Table 3 and Fig. 6. The detailed comparison results are as follows.

a) The proposed model is closer to the observed value than all the comparison models, including WT/SSA/EMD CC-FHS, CEE-FHS, and CEE-CC-PHR, for a 10-min wind speed. For site 1, the developed model has higher prediction accuracy than the other compared models (WT/SSA/EMD CC-FHR, CEE-CC-PHS, and CEEFHS); the MAPE values of the proposed model exhibit improvements of $4.4823 \%$, $4.0087 \%, 1.5747 \%, 0.7818 \%$, and $0.6384 \%$, respectively.

b) In Experiment II, the noise-filtering methods, such as WT, SSA, and EMD, were compared. CEEMDAN significantly outperformed the other noise-filtering methods, as it had higher prediction accuracy. For site 2, compared with the models of WT, SSA, and EMD, the MAPE of the proposed model increased by $3.88 \%$, $3.51 \%$, and $1.26 \%$, respectively. Simultaneously, the MPE values in the range of $(+2.5 \%)$ are more than those of the compared models.

c) By validating the effect of optimizing the input of PSR, CEE-CC-FHS achieved a lower MAPE value than the other models, indicating that the optimal input plays an indispensable role in forecasting chaotic time series. In the experiments for a 10-min wind speed at the three sites, compared with the CEE-FHS model, the MAPE values of the proposed model decreased by $0.7818 \%, 0.8456 \%$, and $0.9317 \%$.

d) Comparison with the PSO algorithm reveals that the FA can better find the optimal model parameters to improve the accuracy of the prediction. The FA not only yields excellent performance for forecasting the wind speed but also outperforms the benchmark in the proposed model. For the three sites, the MPE of the proposed model exhibit in the range of $(+5 \%)$ improvements of $8.08 \%, 9.09 \%$, and $10.61 \%$ compared with the CEE-CC-PHS model, within $\pm 5 \%$ of the measured values.

e) Fig. 6 shows a comparison of the actual values and the values forecasted in the experiment. The proposed prediction model is more accurate than the reference model because more targets converge near the diagonal.

Remark: According to the foregoing analysis, the proposed model shows better performance than the other benchmark models. Consequently, the design of data denoising, the optimal input structure, and the optimization parameters have greatly improved in model prediction. 

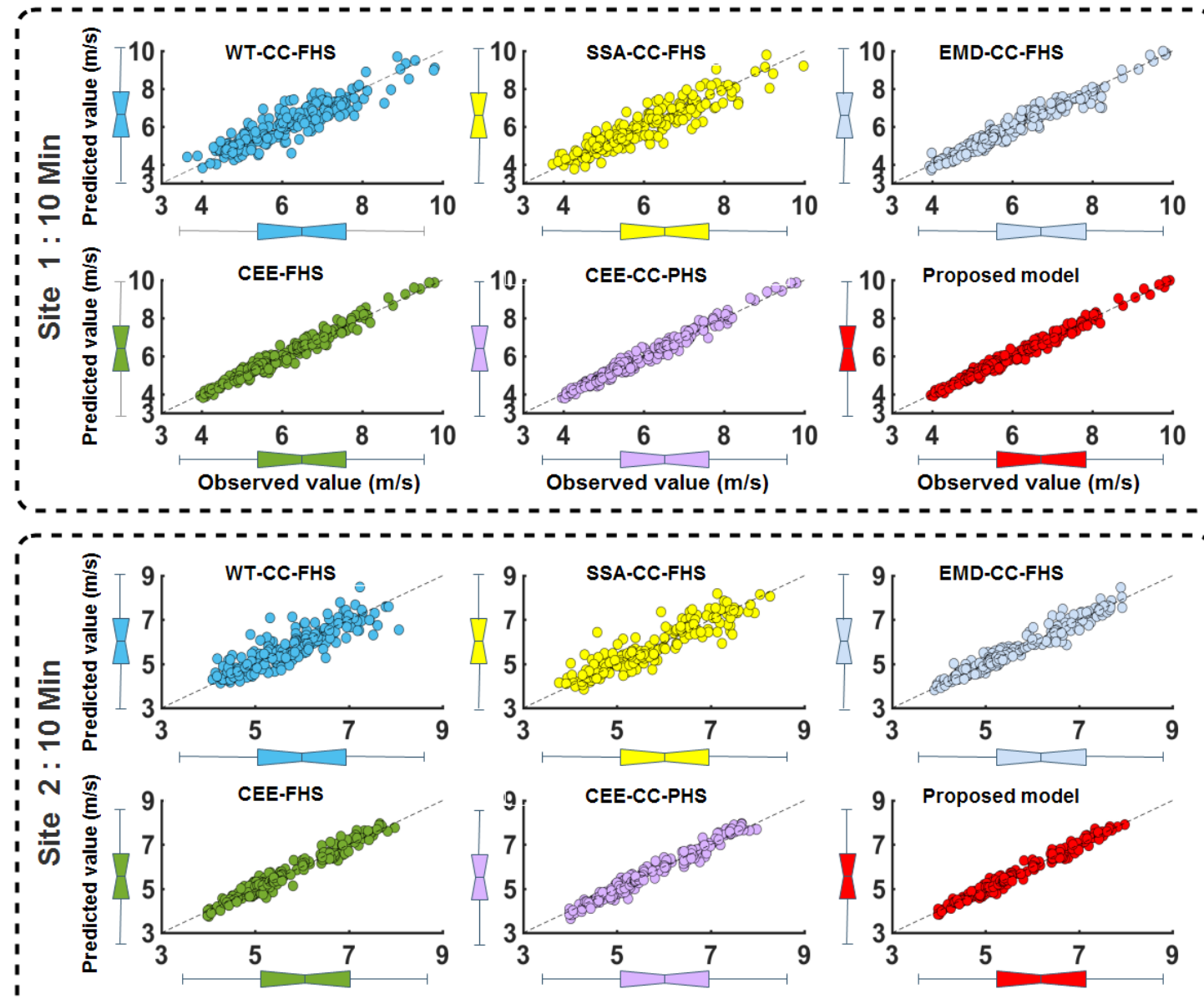

Observed value $(\mathrm{m} / \mathrm{s})$
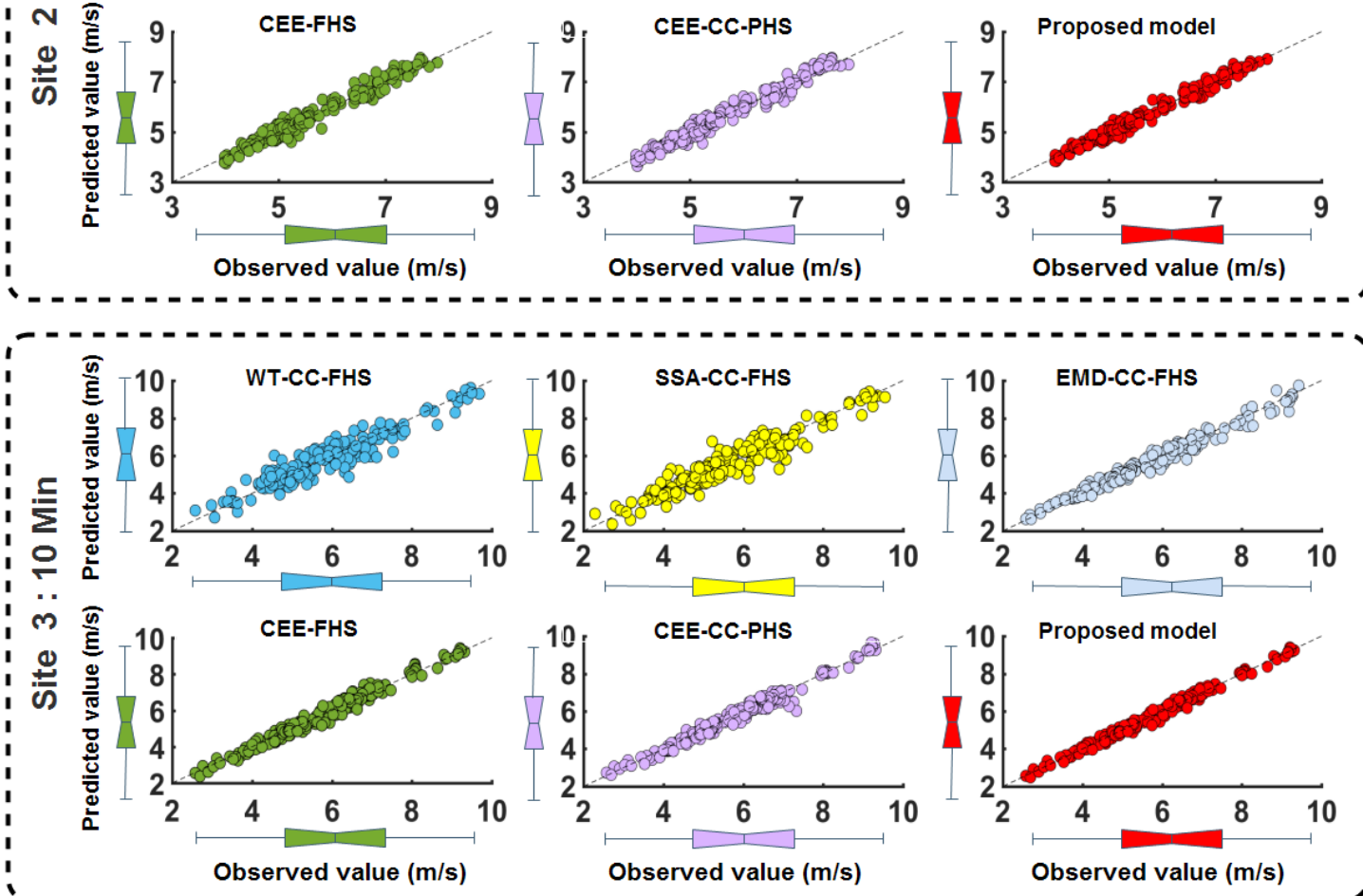

Fig. 6. Comparison of he observed values and the forecasting results for experimental models 
Table 3

Statistical error measures of the six models for 10-Min wind speed at three sites.

\begin{tabular}{|c|c|c|c|c|c|c|c|c|}
\hline \multirow{2}{*}{ Area } & \multirow{2}{*}{ Model } & \multirow{2}{*}{$\begin{array}{c}\text { MAE } \\
(\mathbf{m} / \mathbf{s})\end{array}$} & \multirow{2}{*}{$\begin{array}{l}\text { MSE } \\
(\mathrm{m} / \mathbf{s})\end{array}$} & \multirow{2}{*}{$\begin{array}{c}\text { MAPE } \\
(\%)\end{array}$} & \multicolumn{4}{|c|}{ MPE (\% of errors in each margin) } \\
\hline & & & & & $\pm 2.5 \%$ & $\pm 5 \%$ & $\pm 7.5 \%$ & $\pm 10 \%$ \\
\hline \multirow{6}{*}{ Site1 } & WT-CC-FHS & 0.4121 & 0.2711 & 6.8053 & 20.60 & 44.22 & 66.33 & 76.88 \\
\hline & SSA-CC-FHS & 0.3862 & 0.2472 & 6.3317 & 27.78 & 46.46 & 64.65 & 80.81 \\
\hline & EMD-CC-FHS & 0.2348 & 0.0940 & 3.8977 & 41.21 & 68.84 & 87.44 & 96.48 \\
\hline & CEE-FHS & 0.1894 & 0.0572 & 3.1048 & 49.49 & 77.78 & 93.94 & 98.99 \\
\hline & CEE-CC-PHS & 0.1796 & 0.0494 & 2.9614 & 50.51 & 83.33 & 94.95 & 100.00 \\
\hline & Proposed Model & 0.1413 & 0.0321 & 2.3230 & 62.12 & 91.41 & 99.49 & 100.00 \\
\hline \multirow{6}{*}{ Site2 } & WT-CC-FHS & 0.3422 & 0.2162 & 6.0363 & 30.65 & 54.77 & 70.85 & 81.41 \\
\hline & SSA-CC-FHS & 0.3158 & 0.1828 & 5.6639 & 30.30 & 56.06 & 75.76 & 83.33 \\
\hline & EMD-CC-FHS & 0.1930 & 0.0644 & 3.4196 & 44.22 & 75.88 & 91.96 & 96.98 \\
\hline & CEE-FHS & 0.1678 & 0.0445 & 3.0043 & 50.51 & 84.85 & 94.44 & 98.99 \\
\hline & CEE-CC-PHS & 0.1596 & 0.0404 & 2.8769 & 53.03 & 82.32 & 96.97 & 99.49 \\
\hline & Proposed Model & 0.1207 & 0.0243 & 2.1587 & 65.66 & 91.41 & 98.48 & 100.00 \\
\hline \multirow{6}{*}{ Site3 } & WT-CC-FHS & 0.3627 & 0.2220 & 6.5462 & 28.64 & 50.75 & 62.81 & 77.39 \\
\hline & SSA-CC-FHS & 0.3484 & 0.2122 & 6.4013 & 30.81 & 48.99 & 65.66 & 79.29 \\
\hline & EMD-CC-FHS & 0.1869 & 0.0621 & 3.2421 & 46.73 & 77.39 & 91.96 & 97.99 \\
\hline & CEE-FHS & 0.1746 & 0.0505 & 3.2315 & 48.48 & 74.75 & 91.41 & 98.48 \\
\hline & CEE-CC-PHS & 0.1716 & 0.0542 & 3.1316 & 51.01 & 80.81 & 91.92 & 97.98 \\
\hline & Proposed Model & 0.1252 & 0.0249 & 2.2998 & 62.63 & 91.41 & 97.98 & 100.00 \\
\hline
\end{tabular}




\subsection{Experiment III}

To verify the applicability of the proposed model more comprehensively, a 30-min wind-speed time series for the same three sites was used. The results of the experimental simulations are shown in Tables 4 and 5, from which similar conclusions can be drawn to Experiments I and II. Fig. 7 shows the distribution of the MPE and residual for site 1, and a comparison of the actual and the forecasted values is shown in Fig. 8. We draw the following conclusions.

a) From Table 4, compared with the six models, the proposed model has better forecasting performance as the time interval increases. It can be concluded that that the prediction results of the model are more stable. For site 1, the MPE value of the proposed model is more than that of the comparative model in the range of $(+2.5 \%)$. The MPE values of CEE-CC-ARIMA, CEE-CC-Holt, CEE-CC-GRNN, CEE-CC-SVR, CEE-CC-ARIMA-SVR, and CEE-CC-Holt-GRNN are 13.00\%, $19.10 \%, 30.65 \%, 31.16 \%, 31.31 \%$, and $38.38 \%$, respectively.

b) From Fig. 7, the MPE distribution of the proposed model is more concentrated than those of the other models, and more points are concentrated around the zero point, indicating that more predicted values are close to the actual value. The points in the residual of the proposed model and CEE-CC-FHG are more concentrated in the horizontal zone centered on the origin, indicating that the prediction accuracy is higher than that of the other models.

c) On one hand, CEEMDAN and the $\mathrm{C}-\mathrm{C}$ method can significantly improve the prediction accuracy of the proposed model compared with the benchmark models. On the other hand, the firefly optimization algorithm can improve the forecasting performance, as shown in Table 5 and Fig. 8. For site 1, the proposed model has higher prediction performance than the compared models (WT/SSA/EMD CCFHS, CEE-CC-PHS, and CEE-CC-FHS); the MAPE values of the proposed model exhibit improvements of $2.4045 \%, 7.2375 \%, 1.4267 \%, 0.9519 \%$, and $0.7906 \%$, respectively.

d) From Fig. 8, the WT/SSA/EMD CC-FHC points are more scattered around the diagonal compared with the proposed model. The results indicate that the windspeed time series processed via the CEEMDAN decomposition method combined with PSR can effectively improve the prediction accuracy.

Remark: The test results show that the increase in the wind-speed prediction interval is accompanied by a decrease in accuracy. However, in experimental simulation, the prediction results of the proposed model are better than those of the benchmark model. Therefore, it can be concluded that the proposed model has strong stability and accuracy. 

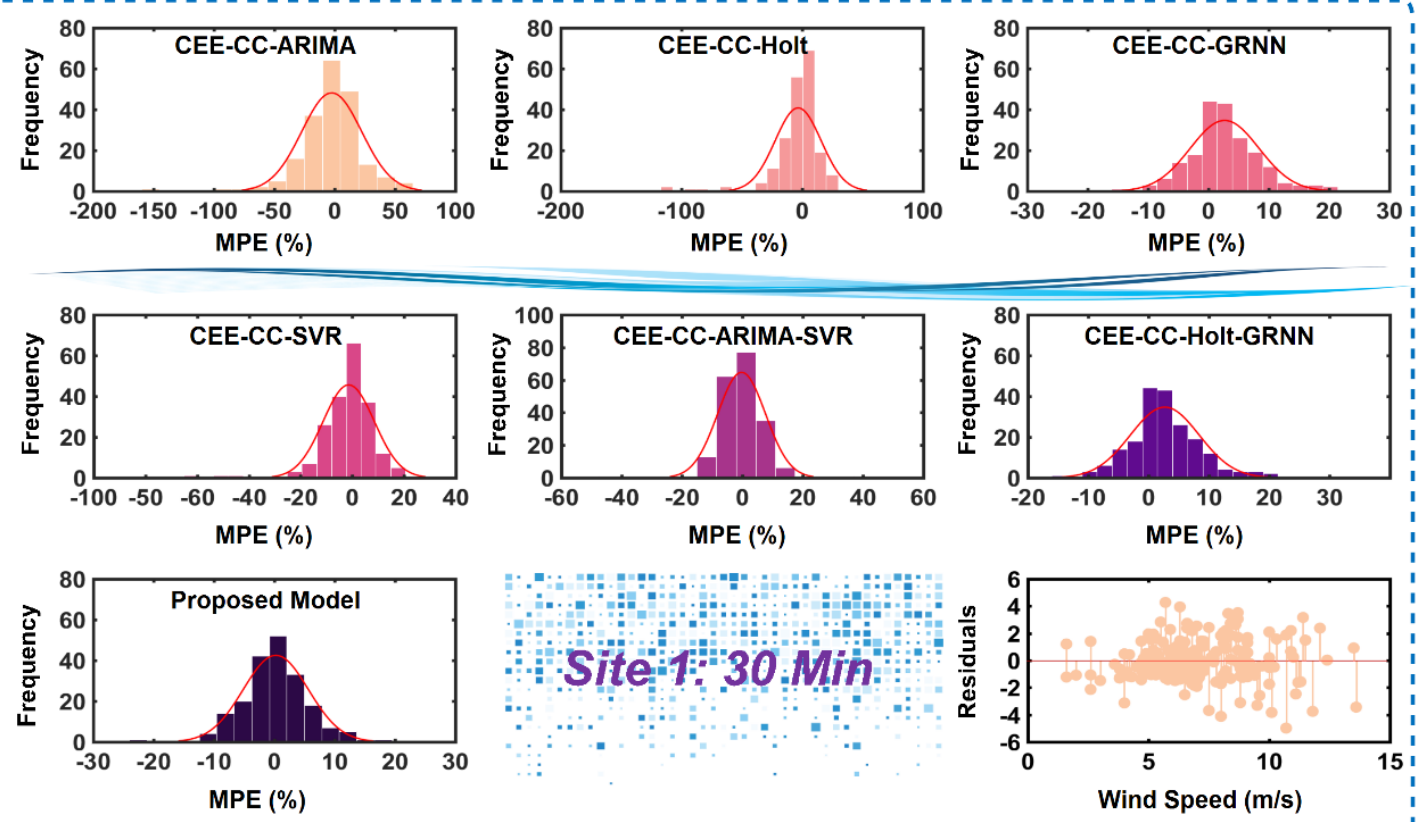

Experiment simulation
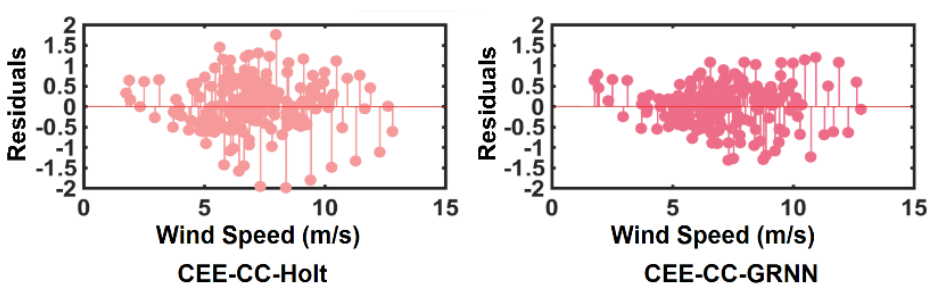

SSE: $78.5261 \quad$ Rsquare: 0.8372

Rmse: 0.6314 Adjrsquare: 0.8363 SSE: $\mathbf{5 1 . 0 0 3 4}$ Rsquare: 0.9290 Rmse: 0.5088 Adjrsquare: 0.9286

Wind Speed $(\mathrm{m} / \mathrm{s})$ CEE-CC-ARIMA

SSE: 472.3343 Rsquare: 0.5529

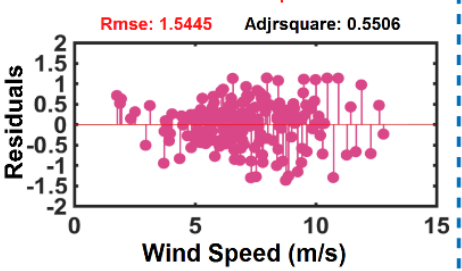

CEE-CC-SVR

SSE: $\mathbf{5 4 . 0 2 4 9}$ Rsquare: 0.9317

Rmse: 0.5237 Adjrsquare: 0.9314
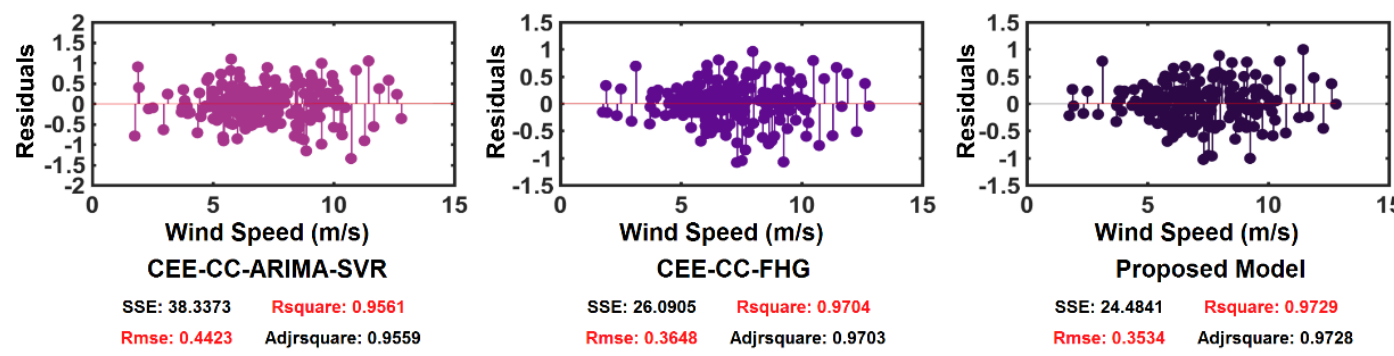

SSE: $\mathbf{3 8 . 3 3 7 3}$ Rsquare: 0.9561

SSE: 26.0905 Rsquare: 0.9704 Rmse: 0.3648 Adjrsquare: 0.9703 Rmse: 0.3534 Adjrsquare: 0.972

Fig. 7. The distribution of MPE and residual for site 1 


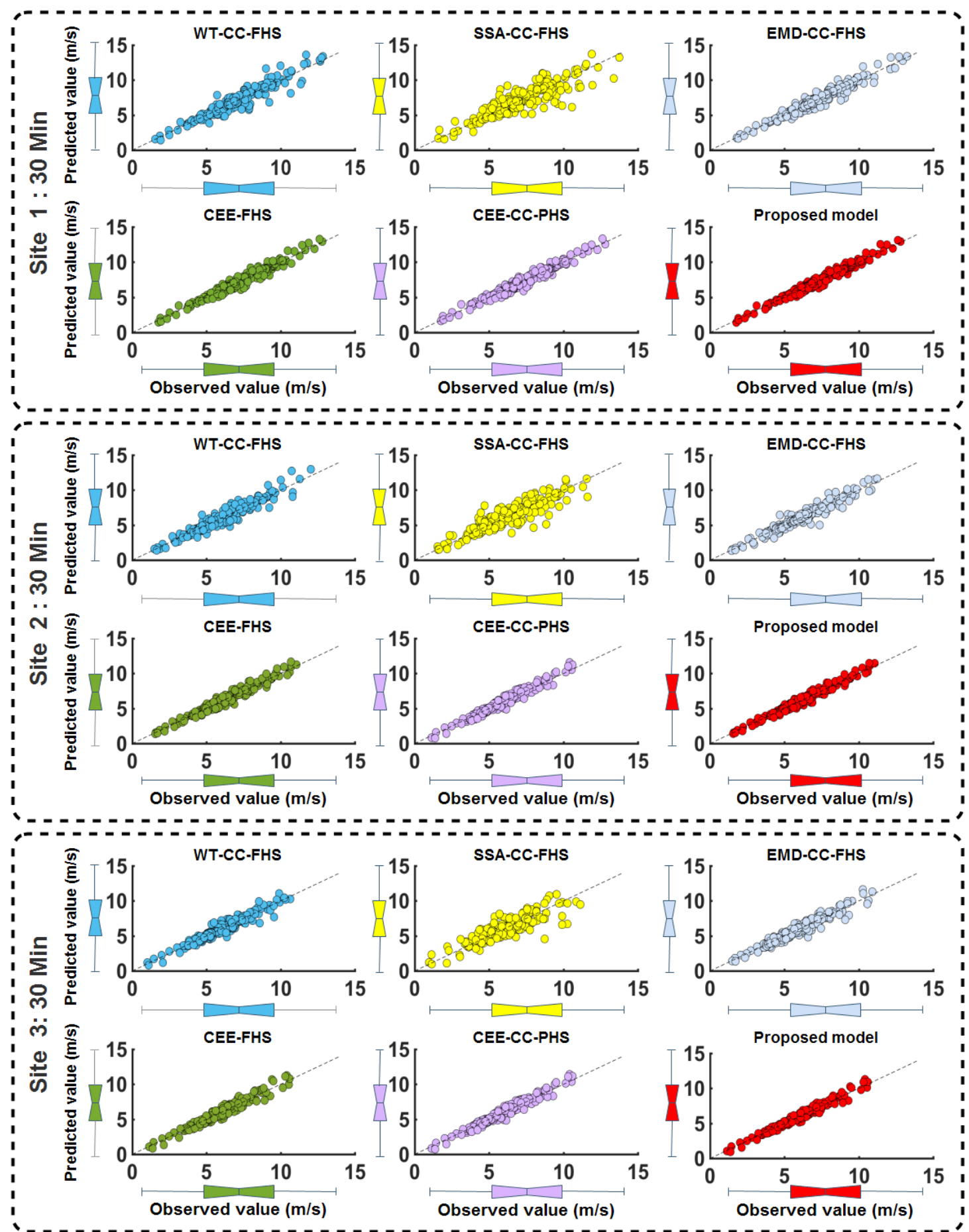

Fig. 8. Comparison of he observed values and the forecasting results for experimental models 
Table 4

Statistical error measures of the seven models for 30-Min wind speed.

\begin{tabular}{|c|c|c|c|c|c|c|c|c|}
\hline \multirow{2}{*}{ Area } & \multirow{2}{*}{ Model } & \multirow{2}{*}{$\begin{array}{l}\text { MAE } \\
(\mathbf{m} / \mathbf{s})\end{array}$} & \multirow{2}{*}{$\begin{array}{l}\text { MSE } \\
(\mathbf{m} / \mathbf{s})\end{array}$} & \multirow{2}{*}{$\begin{array}{c}\text { MAPE } \\
(\%)\end{array}$} & \multicolumn{4}{|c|}{ MPE (\% of errors in each margin) } \\
\hline & & & & & $\pm 2.5 \%$ & $\pm \mathbf{5 \%}$ & $\pm 7.5 \%$ & $\pm \mathbf{1 0 \%}$ \\
\hline \multirow{7}{*}{ Site1 } & CEE-CC-ARIMA & 1.1807 & 2.5805 & 17.3199 & 13.00 & 23.50 & 31.50 & 40.00 \\
\hline & CEE-CC-Holt & 0.6757 & 0.7942 & 11.3996 & 19.10 & 34.67 & 52.26 & 63.82 \\
\hline & CEECC-GRNN & 0.4223 & 0.3071 & 7.0443 & 30.65 & 48.74 & 65.83 & 80.90 \\
\hline & CEE-CC-SVR & 0.4123 & 0.2893 & 6.6013 & 31.16 & 50.25 & 64.32 & 81.41 \\
\hline & CEE-CC-ARIMA-SVR & 0.3461 & 0.1938 & 5.3930 & 31.31 & 57.58 & 77.27 & 89.39 \\
\hline & CEE-CC-FHG & 0.3086 & 0.1593 & 4.7145 & 38.38 & 63.13 & 79.80 & 90.40 \\
\hline & Proposed Model & 0.2708 & 0.1259 & 4.0564 & 40.40 & 69.70 & 85.35 & 93.43 \\
\hline \multirow{7}{*}{ Site2 } & CEE-CC-ARIMA & 1.0554 & 2.0556 & 17.8843 & 13.00 & 20.50 & 29.00 & 39.50 \\
\hline & CEE-CC-Holt & 0.6299 & 0.6104 & 12.1478 & 14.57 & 29.15 & 43.22 & 57.29 \\
\hline & CEECC-GRNN & 0.4219 & 0.2851 & 8.1140 & 21.11 & 43.22 & 60.80 & 75.88 \\
\hline & CEE-CC-SVR & 0.4088 & 0.2618 & 7.3894 & 22.11 & 43.22 & 63.32 & 75.38 \\
\hline & CEE-CC-ARIMA-SVR & 0.3505 & 0.1868 & 6.2453 & 27.27 & 52.02 & 66.67 & 78.79 \\
\hline & CEE-CC-FHG & 0.2961 & 0.1507 & 5.1952 & 36.36 & 56.06 & 74.24 & 85.86 \\
\hline & Proposed Model & 0.2600 & 0.1136 & 4.4386 & 37.37 & 66.67 & 78.79 & 91.92 \\
\hline \multirow{7}{*}{ Site3 } & CEE-CC-ARIMA & 1.0232 & 1.9481 & 18.9920 & 12.50 & 23.00 & 30.50 & 39.00 \\
\hline & CEE-CC-Holt & 0.6345 & 0.6514 & 11.9340 & 12.56 & 30.65 & 42.21 & 53.27 \\
\hline & CEECC-GRNN & 0.4161 & 0.2966 & 8.8052 & 23.12 & 44.22 & 59.30 & 69.85 \\
\hline & CEE-CC-SVR & 0.4088 & 0.2618 & 7.3894 & 22.11 & 43.22 & 63.32 & 75.38 \\
\hline & CEE-CC-ARIMA-SVR & 0.3287 & 0.1886 & 5.7871 & 28.28 & 52.02 & 67.68 & 84.34 \\
\hline & CEE-CC-FHG & 0.2873 & 0.1343 & 5.1527 & 31.31 & 57.58 & 77.78 & 91.92 \\
\hline & Proposed Model & 0.2554 & 0.1105 & 4.5598 & 37.37 & 62.63 & 85.35 & 92.42 \\
\hline
\end{tabular}


Table 5

Statistical error measures of the six models for 30-Min wind speed.

\begin{tabular}{|c|c|c|c|c|c|c|c|c|}
\hline \multirow{2}{*}{ Area } & \multirow{2}{*}{ Model } & \multirow{2}{*}{$\begin{array}{l}\text { MAE } \\
(\mathbf{m} / \mathbf{s})\end{array}$} & \multirow{2}{*}{$\begin{array}{l}\text { MSE } \\
(\mathbf{m} / \mathbf{s})\end{array}$} & \multirow{2}{*}{$\begin{array}{c}\text { MAPE } \\
(\%)\end{array}$} & \multicolumn{4}{|c|}{ MPE (\% of errors in each margin) } \\
\hline & & & & & $\pm 2.5 \%$ & $\pm 5 \%$ & $\pm 7.5 \%$ & $\pm \mathbf{1 0 \%}$ \\
\hline \multirow{6}{*}{ Site1 } & WT-CC-FHS & 0.4536 & 0.4699 & 6.4609 & 36.68 & 54.77 & 69.85 & 78.39 \\
\hline & SSA-CC- FHS & 0.7804 & 1.1958 & 11.2939 & 20.20 & 35.35 & 46.97 & 57.07 \\
\hline & EMD-CC- FHS & 0.3809 & 0.2671 & 5.4831 & 33.17 & 56.78 & 73.37 & 84.42 \\
\hline & CEE- FHS & 0.3351 & 0.1922 & 5.0083 & 32.83 & 60.10 & 76.26 & 86.87 \\
\hline & CEE-CC- PHS & 0.3161 & 0.1722 & 4.8470 & 37.88 & 59.09 & 79.29 & 86.87 \\
\hline & Proposed Model & 0.2708 & 0.1259 & 4.0564 & 40.40 & 69.70 & 85.35 & 93.43 \\
\hline \multirow{6}{*}{ Site2 } & WT-CC-FHS & 0.4194 & 0.3447 & 7.0936 & 17.09 & 33.17 & 50.25 & 75.38 \\
\hline & SSA-CC- FHS & 0.6921 & 0.8928 & 11.7166 & 13.13 & 29.29 & 44.95 & 55.05 \\
\hline & EMD-CC- FHS & 0.3501 & 0.2520 & 5.9175 & 33.17 & 58.79 & 72.86 & 83.42 \\
\hline & CEE- FHS & 0.3160 & 0.1666 & 5.3843 & 29.80 & 58.08 & 73.23 & 84.34 \\
\hline & CEE-CC- PHS & 0.3046 & 0.1522 & 5.6108 & 29.80 & 53.03 & 75.25 & 85.35 \\
\hline & Proposed Model & 0.2600 & 0.1136 & 4.4386 & 37.37 & 66.67 & 78.79 & 91.92 \\
\hline \multirow{6}{*}{ Site3 } & WT-CC-FHS & 0.3439 & 0.2241 & 6.1986 & 31.16 & 53.77 & 69.35 & 81.41 \\
\hline & SSA-CC- FHS & 0.6892 & 0.8641 & 12.5879 & 14.65 & 27.27 & 41.41 & 50.51 \\
\hline & EMD-CC- FHS & 0.3588 & 0.2218 & 6.2247 & 25.63 & 46.73 & 67.34 & 84.42 \\
\hline & CEE- FHS & 0.3343 & 0.1829 & 5.9659 & 27.27 & 53.03 & 71.21 & 83.33 \\
\hline & CEE-CC-PHS & 0.3126 & 0.1569 & 5.7798 & 25.25 & 52.02 & 72.73 & 85.35 \\
\hline & Proposed Model & 0.2554 & 0.1105 & 4.5598 & 37.37 & 62.63 & 85.35 & 92.42 \\
\hline
\end{tabular}




\section{Analysis and discussions}

This section presents a detailed discussion of the proposed model. Additionally, the optimization performance of the firefly algorithm is verified by comparison with other optimization algorithms. Finally, a comparative analysis of the relevant literature is made. The validity of the experimental results was analyzed and verified by a hypothesis test. Finally, this study was compared with related literature.

\subsection{Comparative analysis}

In the previous three experiments, it was proven that CEE-CC-FHS is far superior to the compared models. To comprehensively test the superiority of the model, one statistical method and three intelligent algorithms are considered to compare the windspeed forecasting accuracy. These algorithms are the ARIMA, the backpropagation neural network (BPNN), GRNN, and ELM, respectively. According to the results shown in Table 6, the following conclusions are drawn.

Compared with the three intelligent algorithms, the proposed model has superior and stable prediction performance. Thus, the proposed model provides an effective forecasting method for wind speed. For a 10-min wind speed at site 1, the proposed model obtains the best forecasting performance. Compared with the ARIMA, BPNN, GRNN, and ELM, the MAPEs of the proposed model exhibit improvements of $7.4216 \%, 5.1233 \%, 6.8001 \%$, and $4.9496 \%$, respectively. Similarly, for a 30-min wind speed at site 1, the MAPEs of the proposed model compared with the ARIMA, BPNN, GRNN, and ELM exhibit improvements of $15.0258 \%, 8.9732 \%, 9.2045 \%$, and $8.7387 \%$, respectively. According to the comparison error between the proposed model and the three prediction models, the data preprocessing techniques combined with linear and nonlinear prediction frameworks can significantly improve the prediction accuracy. 
Table 6

Comparison of prediction performances of four other models and proposed model.

\begin{tabular}{|c|c|c|c|c|c|c|c|}
\hline \multirow{2}{*}{ Model } & \multirow{2}{*}{ Indices } & \multicolumn{3}{|c|}{ 10-Min } & \multicolumn{3}{|c|}{ 30-Min } \\
\hline & & Site 1 & Site 2 & Site 3 & Site 1 & Site 2 & Site 3 \\
\hline \multirow{5}{*}{ ARIMA } & $\operatorname{MAE}(\mathrm{m} / \mathrm{s})$ & 0.5997 & 0.8419 & 0.6345 & 1.3042 & 1.2281 & 1.1702 \\
\hline & $\operatorname{MSE}(\mathrm{m} / \mathrm{s})$ & 0.5635 & 1.2116 & 0.6187 & 2.8842 & 2.4948 & 2.3833 \\
\hline & MAPE (\%) & 9.7446 & 15.0181 & 11.6620 & 19.0822 & 20.6847 & 21.7168 \\
\hline & $\operatorname{MPE}( \pm 5 \%)$ & 30.00 & 23.00 & 27.50 & 16.00 & 13.50 & 12.00 \\
\hline & MPE $( \pm 10 \%)$ & 59.50 & 44.00 & 48.00 & 33.50 & 24.00 & 29.00 \\
\hline \multirow{5}{*}{ BPNN } & $\operatorname{MAE}(\mathrm{m} / \mathrm{s})$ & 0.4474 & 0.3844 & 0.3772 & 0.8698 & 0.8132 & 0.7511 \\
\hline & $\operatorname{MSE}(\mathrm{m} / \mathrm{s})$ & 0.3112 & 0.2657 & 0.2314 & 1.3879 & 1.1624 & 1.0198 \\
\hline & MAPE $(\%)$ & 7.4463 & 6.9510 & 7.0167 & 13.0296 & 14.2048 & 14.2712 \\
\hline & $\operatorname{MPE}( \pm 5 \%)$ & 41.50 & 46.00 & 44.50 & 31.00 & 26.00 & 26.50 \\
\hline & $\operatorname{MPE}( \pm 10 \%)$ & 72.50 & 80.50 & 78.00 & 53.50 & 48.00 & 49.50 \\
\hline \multirow{5}{*}{ GRNN } & $\operatorname{MAE}(\mathrm{m} / \mathrm{s})$ & 0.5592 & 0.4793 & 0.5385 & 0.8741 & 0.9217 & 0.7615 \\
\hline & $\operatorname{MSE}(\mathrm{m} / \mathrm{s})$ & 0.5028 & 0.3807 & 0.4662 & 1.3991 & 1.4194 & 1.0402 \\
\hline & MAPE (\%) & 9.1231 & 8.7769 & 10.9159 & 13.2609 & 17.0663 & 15.4501 \\
\hline & MPE $( \pm 5 \%)$ & 33.50 & 36.50 & 39.50 & 31.50 & 20.50 & 28.50 \\
\hline & MPE $( \pm 10 \%)$ & 59.50 & 65.50 & 58.50 & 52.00 & 41.00 & 48.00 \\
\hline \multirow{5}{*}{ ELM } & $\mathrm{MAE}(\mathrm{m} / \mathrm{s})$ & 0.4453 & 0.3615 & 0.3685 & 0.8693 & 0.7858 & 0.7413 \\
\hline & $\operatorname{MSE}(\mathrm{m} / \mathrm{s})$ & 0.3163 & 0.2477 & 0.2272 & 1.4046 & 1.0967 & 1.0117 \\
\hline & MAPE (\%) & 7.2726 & 6.4389 & 6.8388 & 12.7951 & 13.6257 & 13.8748 \\
\hline & $\operatorname{MPE}( \pm 5 \%)$ & 44.00 & 51.50 & 49.50 & 30.50 & 27.00 & 26.00 \\
\hline & MPE $( \pm 10 \%)$ & 73.00 & 79.00 & 76.00 & 53.50 & 49.50 & 50.00 \\
\hline \multirow{5}{*}{$\begin{array}{c}\text { Proposed } \\
\text { Model }\end{array}$} & $\operatorname{MAE}(\mathrm{m} / \mathrm{s})$ & 0.1413 & 0.1207 & 0.1252 & 0.2708 & 0.2600 & 0.2554 \\
\hline & $\operatorname{MSE}(\mathrm{m} / \mathrm{s})$ & 0.0321 & 0.0243 & 0.0249 & 0.1259 & 0.1136 & 0.1105 \\
\hline & MAPE (\%) & 2.3230 & 2.1587 & 2.2998 & 4.0564 & 4.4386 & 4.5598 \\
\hline & $\operatorname{MPE}( \pm 5 \%)$ & 91.41 & 91.41 & 91.41 & 69.70 & 66.67 & 62.63 \\
\hline & MPE $( \pm 10 \%)$ & 100.00 & 100.00 & 100.00 & 93.43 & 91.92 & 92.42 \\
\hline
\end{tabular}

\subsection{Evaluate performance of optimization algorithm}

In the previous experiments, the proposed model was compared with the model (CEE-CC-PHS) optimized by the PSO algorithm. The results show that the FA can improve the predictive performance of the model. To more clearly demonstrate the performance of the FA, three typical test functions - Sphere, Rastrigin, and Ackleywere used for verification. The variable fields and function formulas of the test function are shown in Table 7.

Table 7

Three test functions.

\begin{tabular}{cccc}
\hline Function & $\begin{array}{c}\text { Variable } \\
\text { domain }\end{array}$ & $\begin{array}{c}\text { Optimum } \\
\text { value }\end{array}$ & Test function \\
\hline Sphere & {$[-1,1]$} & 0 & $\sum_{i=1}^{d} x_{i}^{2}$ \\
Ackley & {$[-10,10]$} & 0 & $-20 \exp \left[-0.2 \sqrt{\frac{1}{d} \sum_{i=1}^{d} x_{i}}\right]-\exp \left[\frac{1}{d} \sum_{i=1}^{d} \cos \left(2 \pi x_{i}\right)\right]+20+e$ \\
Rastrigin & {$[-5,5]$} & 0 & $\sum_{i=1}^{d}\left(x_{i}^{2}-10\left(2 \pi x_{i}\right)+10\right)$ \\
\hline
\end{tabular}

Additionally, optimization algorithms - the PSO algorithm, the cuckoo search algorithm, and other heuristics algorithms, i.e., the bat algorithm (BA) and flower 
pollination algorithm - are selected for comparative analysis. Fig. 9 shows the variation of the fitness with the increase of the number of iterations for different numbers of dimensions. The test results for 30 runs of the FA and the other algorithms are presented in Table 8.

For the Sphere function, the fitness of the five optimization algorithms tends to zero after 200 iterations, regardless of whether the number of dimensions is 10 or 20 . However, as shown in Fig. 9, the convergence speed of the FA is better than that of the other four algorithms. When the dimension is 10, the minimum, average, and standard deviation of the FA test results are 6.65E-08, 1.21E-06, and 1.89E-06, respectively. The optimization results show that the FA has the best search ability among the algorithms. For the Ackley function, the test results of different algorithms differ significantly, as shown in Fig. 9. When the dimension is 10, the search results of the FA are significantly better than those of the other four algorithms, and the minimum value of the fitting in 200 iterations is 0.0049 . When the dimension is 20 , the search results of the FA and BA are better than those of the other three algorithms. In addition, the minimum value of the FA is 0.0428 , and the FA has the lowest fitness value. For the Rastrigin function, the convergence speed of the FA in 200 iterations does not differ significantly from those of the other algorithms. For dimensions 10 and 20, the minimum values of the FA are 3.6810 and 23.2860, respectively. The results show that the FA can find the lowest fitness values.

The optimization results for different test functions in different dimensions indicate that FA has a more accurate and stable search capability than the other optimization algorithms. Specifically, the maximum, minimum, average, and standard deviation are lower for the FA than for the other algorithms. Therefore, the search performance and verification results of the FA algorithm are superior to those of the other algorithms.

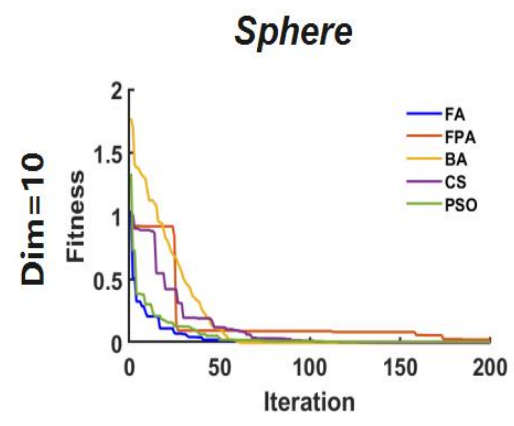

Ackley

Rastrigin
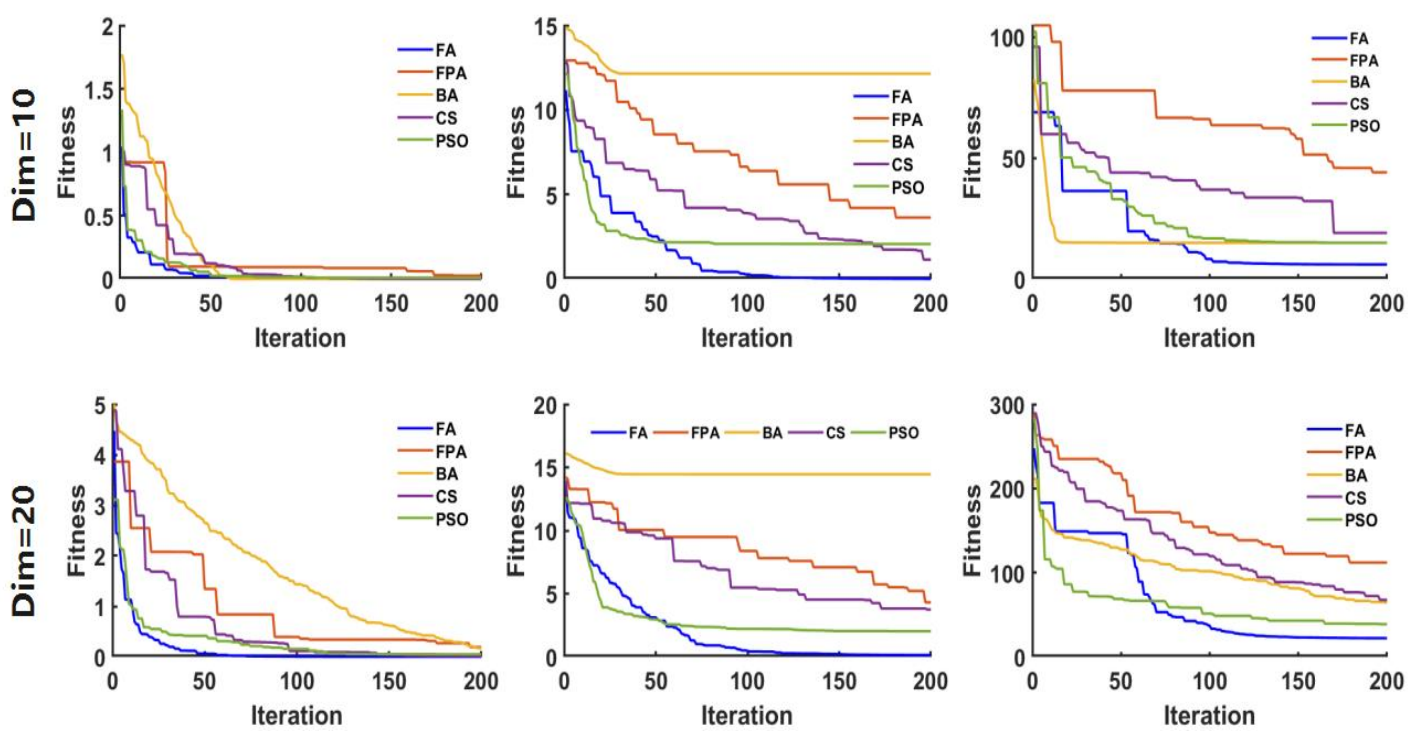

Fig. 9. Test results of FA and other algorithms. 
Table 8

Test results of 30 trials of FA and other algorithms.

\begin{tabular}{|c|c|c|c|c|c|c|}
\hline $\begin{array}{c}\text { Function } \\
\text { name }\end{array}$ & Dim & Algorithm & $\begin{array}{c}\text { Max } \\
\text { value } \\
\end{array}$ & $\begin{array}{c}\text { Min } \\
\text { value }\end{array}$ & $\begin{array}{c}\text { Average } \\
\text { value }\end{array}$ & $\begin{array}{l}\text { Standard } \\
\text { deviation }\end{array}$ \\
\hline \multirow{5}{*}{ Sphere } & \multirow{5}{*}{10} & PSO & $1.64 \mathrm{E}-02$ & $1.20 \mathrm{E}-03$ & $6.11 \mathrm{E}-03$ & $4.33 \mathrm{E}-03$ \\
\hline & & $\mathrm{CS}$ & $3.08 \mathrm{E}-04$ & $4.67 \mathrm{E}-05$ & $1.55 \mathrm{E}-04$ & $7.25 \mathrm{E}-05$ \\
\hline & & BA & $1.92 \mathrm{E}-04$ & $1.14 \mathrm{E}-04$ & $1.44 \mathrm{E}-04$ & $2.57 \mathrm{E}-05$ \\
\hline & & FPA & 7.74E-02 & $2.47 \mathrm{E}-02$ & $4.03 \mathrm{E}-02$ & $1.56 \mathrm{E}-02$ \\
\hline & & FA & $6.22 \mathrm{E}-06$ & $6.65 E-08$ & $1.21 \mathrm{E}-06$ & 1.89E-06 \\
\hline \multirow{5}{*}{ Sphere } & \multirow{5}{*}{20} & PSO & 0.0851 & 0.0106 & 0.0435 & 0.0310 \\
\hline & & $\mathrm{CS}$ & 0.0385 & 0.0121 & 0.0196 & 0.0083 \\
\hline & & BA & 0.7408 & 0.0123 & 0.1449 & 0.2235 \\
\hline & & FPA & 0.3588 & 0.1485 & 0.2595 & 0.0560 \\
\hline & & FA & 0.0116 & 0.0019 & 0.0077 & 0.0028 \\
\hline \multirow{5}{*}{ Ackley } & \multirow{5}{*}{10} & PSO & 2.6123 & 1.6681 & 2.1834 & 0.3315 \\
\hline & & $\mathrm{CS}$ & 1.7050 & 0.8431 & 1.3319 & 0.2438 \\
\hline & & BA & 12.5431 & 10.1768 & 11.3870 & 0.7874 \\
\hline & & FPA & 5.2881 & 3.4698 & 4.5021 & 0.5844 \\
\hline & & FA & 0.0154 & 0.0049 & 0.0089 & 0.0037 \\
\hline \multirow{5}{*}{ Ackley } & \multirow{5}{*}{20} & PSO & 4.0309 & 2.1687 & 2.9099 & 0.6136 \\
\hline & & $\mathrm{CS}$ & 3.8508 & 3.3545 & 3.5989 & 0.2080 \\
\hline & & $\mathrm{BA}$ & 15.1835 & 13.6162 & 14.4169 & 0.5778 \\
\hline & & FPA & 7.2390 & 5.3972 & 6.2940 & 0.6090 \\
\hline & & FA & 0.1570 & 0.0428 & 0.1075 & 0.0306 \\
\hline \multirow{5}{*}{ Rastrigin } & \multirow{5}{*}{10} & PSO & 18.8020 & 7.5053 & 12.4701 & 3.5539 \\
\hline & & $\mathrm{CS}$ & 18.7541 & 10.5480 & 15.8923 & 2.5198 \\
\hline & & $\mathrm{BA}$ & 17.9941 & 4.1491 & 11.3233 & 3.8493 \\
\hline & & FPA & 40.4292 & 24.0871 & 35.5402 & 4.9954 \\
\hline & & FA & 9.3277 & 3.6810 & 7.4864 & 1.8292 \\
\hline \multirow{5}{*}{ Rastrigin } & \multirow{5}{*}{20} & PSO & 82.8993 & 43.7370 & 60.8321 & 13.8520 \\
\hline & & $\mathrm{CS}$ & 94.7210 & 58.4668 & 76.5177 & 11.2896 \\
\hline & & BA & 88.3268 & 33.9320 & 67.9996 & 18.1168 \\
\hline & & FPA & 135.4307 & 103.6248 & 119.0780 & 9.1622 \\
\hline & & FA & 53.9004 & 23.2860 & 42.0311 & 8.4956 \\
\hline
\end{tabular}

\subsection{Test the validity of the model}

This section is aimed at comparing the six other models (CEEN-CC-Holt, CEE-CCARIMA, CEEN-CC-GRNN, CEE-CC-SVR, CEE-CC-ARIMA-SVR, and CEE-CCHolt-GRNN) through the error criteria (listed in Table 9). According to the results shown in Table 10, the performance of the forecasting model is compared and analyzed as follows.

With regard to the wind-speed forecasting accuracy, the prediction performance of the hybrid model is better than that of the benchmark model. Table 8 indicates that the value of the percentage increase decreases gradually as the interval of data collection increases. For the proposed model vs. CEE-CC-FHG for a 10-min wind speed, the $P_{M A P E}$ values for sites 1,2 , and 3 are $34.1942 \%, 27.1784 \%$, and $25.2083 \%$, respectively. According to the comparison order (i.e., compared with CEE-CC-ARIMA, CEE-CCHolt, CEE-CC-GRNN, CEE-CC-SVR, CEE-ARIMA-SVR, and CEE-CC-FHG), the percentage improvement of the error criterion gradually decreases, which indicates that the prediction performance of the compared model increases. This also signifies that the proposed model has higher prediction accuracy than the other models. The 
improvement in the prediction accuracy of the comparison model to a certain level is accompanied by a decrease in the model prediction improvement.

Table 9

Three improvement percentage of criteria.

\begin{tabular}{ccc}
\hline Metric & Definition & Equation \\
\hline$P_{M A E}$ & The improvement percentages of MAE & $P_{M A E}=\left|\frac{M A E_{1}-M A E_{2}}{M A E_{1}}\right|$ \\
$P_{M S E}$ & The improvement percentages of MSE & $P_{M S E}=\left|\frac{M S E_{1}-M S E_{2}}{M S E_{1}}\right|$ \\
$P_{M A P E}$ & The improvement percentages of MAPE & $P_{M A P E}=\left|\frac{M A P E_{1}-M A P E_{2}}{M A P E_{1}}\right|$ \\
\hline
\end{tabular}

Table 10

Improvement percentages among the proposed model and the six models.

\begin{tabular}{|c|c|c|c|c|c|c|c|}
\hline \multirow{2}{*}{ Model } & \multirow{2}{*}{ Indices } & \multicolumn{3}{|c|}{ 10-Min } & \multicolumn{3}{|c|}{ 30-Min } \\
\hline & & site 1 & site 2 & site 3 & site 1 & site 2 & site 3 \\
\hline \multirow{3}{*}{$\begin{array}{c}\text { Proposed vs. } \\
\text { CEE-CC-ARIMA }\end{array}$} & & & & & 77.0674 & & \\
\hline & & & 96.9 & 19 & 95.1228 & & 94.3285 \\
\hline & & 68.9 & 82.5 & 78.6 & 76.5 & & 75.991 \\
\hline \multirow{3}{*}{$\begin{array}{l}\text { Proposed vs. } \\
\text { CEE-CC-Holt }\end{array}$} & & & & & & & \\
\hline & & & 3 & 14 & & & 83.0383 \\
\hline & & 66. & 7 & 67.5 & 62 & & 61.7916 \\
\hline \multirow{3}{*}{$\begin{array}{c}\text { Proposed vs. } \\
\text { CEE-CC-GRNN }\end{array}$} & & & & 40.2 & 53 & & 38.6276 \\
\hline & & 78.3 & 73. & 64.8 & 59. & & 62.7546 \\
\hline & & 57.8 & 51.9 & & & & 48.2148 \\
\hline \multirow{3}{*}{$\begin{array}{l}\text { Proposed vs. } \\
\text { CEE-CC-SVR }\end{array}$} & & 52.1905 & 29.7 & 33.4 & 34.3 & & 37.5328 \\
\hline & & 75.8934 & 49.68 & 59.4463 & 56.4918 & & 57.7927 \\
\hline & & 52.347 & 29.3 & 35.4768 & 38.5 & & 38.2933 \\
\hline \multirow{3}{*}{$\begin{array}{l}\text { Proposed vs. } \\
\text { CEE-CC- } \\
\text { ARIMA-SVR }\end{array}$} & & 44.36 & & & 21.7 & & 22.2986 \\
\hline & & & & & & & 41.4087 \\
\hline & & 43.2093 & 20.1 & 20.7138 & 24.7838 & 28.9297 & 21.2074 \\
\hline \multirow{3}{*}{$\begin{array}{l}\text { Proposed vs. } \\
\text { CEE-CC-FHG }\end{array}$} & & 33.1186 & & 24.2376 & 12.2575 & 12.1977 & 11.0964 \\
\hline & $\mathrm{P}_{\mathrm{MSE}}$ & 52.181 & 38.9412 & 37.4163 & 21.0075 & 24.6318 & 17.7311 \\
\hline & $\mathrm{P}_{\mathrm{MAPE}}$ & 34.1942 & 27.1784 & 25.2083 & 13.9598 & 14.5648 & 11.5069 \\
\hline
\end{tabular}

\subsection{Diebold-Mariano test}

The Diebold-Mariano (DM) test was performed to assess the forecasting abilities of the proposed model and the comparison models [41]. The null hypothesis $H_{0}$ and the alternative hypothesis $H_{1}$ for the DM test are expressed as follows:

$$
\begin{gathered}
\left.H_{0}: E\left[L\left(\text { error }_{l}^{1}\right)\right]=E\left[L\left(\text { error }_{l}^{2}\right)\right]\right] \\
\left.H_{1}: E\left[L\left(\text { error }_{l}^{1}\right)\right] \neq E\left[L\left(\text { error }_{l}^{2}\right)\right]\right] .
\end{gathered}
$$

The DM test statistics can be obtained as

$$
D M=\frac{\sum_{i=1}^{n}\left(L\left(\text { error }_{i}^{1}\right)-L\left(\text { error }_{i}^{2}\right)\right) / n}{\sqrt{S^{2} / n}} s^{2},
$$


where $s^{2}$ represents an estimation for the variance of $d_{i}=L\left(\varepsilon_{i}^{1}\right)-L\left(\varepsilon_{i}^{2}\right)$. When the DM statistic under the significant level of $\alpha$ falls in the interval $\left[-Z_{\alpha / 2}, Z_{\alpha / 2}\right]$, the null hypothesis $H_{0}$ is accepted. This means that the predicted performance is not significantly different between the proposed model and the compared model.

Table 11 compares the five models (WT-CC-FHS, SSA-CC-FHS, EMD-CC- FHS, CEE-FHS, and CEE-CC-PHS) under the confidence level of $1 \%$. The minimum value of $|\mathrm{DM}|$ is 2.9036 , which is greater than $Z_{0.01 / 2}=2.58$. The results show that the probability difference between the proposed model and the compared models is $99 \%$. The DM test proves not only the effectiveness of the experiment but also the superiority of the proposed model.

Table 11

The results of Diebold-Mariano test.

\begin{tabular}{ccccc}
\hline DM test & Average value & Site1 & Site2 & Site3 \\
\hline & WT-CC- FHS & $8.7471^{*}$ & $6.6088^{*}$ & $8.1942^{*}$ \\
& SSA-CC- FHS & $8.6197^{*}$ & $6.0762^{*}$ & $7.3828^{*}$ \\
10-Min & EMD-CC- FHS & $4.9286^{*}$ & $5.2528^{*}$ & $5.1451^{*}$ \\
& CEE-FHS & $7.7355^{*}$ & $6.4175^{*}$ & $7.3282^{*}$ \\
& CEE-CC-PHS & $4.9232^{*}$ & $5.1551^{*}$ & $2.9036^{*}$ \\
\hline \multirow{5}{*}{ 30-Min } & WT-CC- FHS & $4.7042^{*}$ & $5.7147^{*}$ & $4.0590^{*}$ \\
& SSA-CC- FHS & $6.4104^{*}$ & $6.3993^{*}$ & $5.8269^{*}$ \\
& EMD-CC- FHS & $5.2752^{*}$ & $3.9622^{*}$ & $5.1214^{*}$ \\
& CEE-FHS & $6.4708^{*}$ & $7.3642^{*}$ & $7.0861^{*}$ \\
\hline
\end{tabular}

Note: ${ }^{* 1 \%}$ significance level.

\subsection{Comparison of the related literature}

Prediction methods based on decomposition techniques have been widely applied to the actual wind speed $[42,43]$. Wang et al. [44] reported that the model established by the EMD-based prediction algorithm may not be suitable for the updated time series in actual wind-speed prediction and proposed an approximate prediction model based on EMD. With regard to the decomposition method, the processing idea of this study is similar to that of Wang [44]; that is, the most frequent $I M F_{1}$ is removed from the original time series, and the approximate time series is obtained for the next analysis. When the original time series is decomposed with the newly obtained data, the proportion of the $I M F_{1}$ in the original wind-speed time series is small; thus, the influence of the $I M F_{1}$ change on the time series is not significant.

Additionally, in this study, reconstruction technology is used to select features, reconstruct the phase space of the approximate time series to determine the delay time and dimension of the time series, and extend one-dimensional time series to a matrix time series. The matrix time series is used as an input of the model to more clearly show the variation of the time series [45]. According to the literature [46], C-C technology has achieved satisfactory forecasting results in determining the input form of the model. However, in these studies, the model did not capture well the linear and nonlinear factors of the time series from the optimal input determined by the $\mathrm{C}-\mathrm{C}$ method. Accordingly, in the present study, a "linear and nonlinear" modeling framework was developed to comprehensively excavate the information in the time series. Furthermore, the firefly optimization algorithm is an effective technique that provides optimal model parameters to improve the prediction performance of the model. The performance of the firefly optimization algorithm is described in detail in [47]. Additionally, the experimental results show that the data denoising technique CEEMDAN provides a 
significant model prediction performance improvement. A similar conclusion was drawn in [48]; however, that model does not comprehensively capture the information in the time series after CEEMDAN is used to remove the interference factors. Moreover, in this study, CEEMDAN employed PSR to maximize the mining of hidden real-time information in the time series.

\section{Conclusions}

Accurate wind-speed prediction is of great help for the improvement of wind energy efficiency. However, the wind-speed time series contain many disturbing factors, which pose a severe challenge to accurate wind-speed forecasting. Abundant work has been devoted to improving the performance of models for forecasting wind speed. Therefore, it is very valuable to develop a model with optimized performance for wind-speed prediction.

In this paper, the CEE-CC-FHS model is proposed for chaotic series forecasting. The importance of data preprocessing was verified, which provided as much information as possible for the predictive model. CEEMDAN was developed to eliminate interference components from the original data, and PSR was proven to be a feasible method for capturing the information of time-series fluctuation. The matrix time series can provide more information contained in the time series for the forecasting. Thus, changing wind speeds can be converted into chaotic systems for research.

Experiments indicated that the proposed model removes the interference factors of the chaotic time series and provides valuable predictive information while having the ability to capture linear and nonlinear modes, especially for a chaotic time series characterized by complexity and irregularity. In Experiment I, the average MAPE of the proposed model compared with other models (CEE-CC-ARIMA, CEE-CC-Holt, CEE-CC-GRNN, CEE-CC-SVR, CEE-CC-ARIMA-SVR, and CEE-CC-Holt-GRNN) exhibited improvements of $7.9324 \%, 4.8683 \%, 2.4176 \%, 1.5716 \%, 0.9705 \%$, and $0.9293 \%$, respectively. In Experiment II, the average MAPE of the designed forecasting architecture exhibited improvements of $4.2021 \%, 3.8718 \%, 1.2593 \%, 0.8530 \%$, and $0.7295 \%$ compared with the benchmark models WT/SSA/EMD CC-FHS, CEE-FHS, and CEE-CC-PHS, respectively. In Experiment III, the average MAPE of the proposed model was better than that of the compared models.

The results indicate that the wind speed at different sites can be effectively predicted by the proposed model. Furthermore, the experimental results based on statistical criteria indicate that the proposed model exhibits better forecasting performance than other models. Overall, the model improves the accuracy of wind-speed prediction and provides a new feasible solution for wind-speed prediction and rational power-grid allocation.

\section{Acknowledgements}

This research was supported by the National Natural Science Foundation of China (Grant No. 71573034). All the authors do not have any possible conflicts of interest. 


\section{Appendix A}

\section{List of abbreviations}

\begin{tabular}{|c|c|c|c|}
\hline Nomenclature & & $\begin{array}{l}\text { ARIMA } \\
\text { PSR }\end{array}$ & $\begin{array}{l}\text { Autoregressive Integrated Moving Average } \\
\text { predicted value of the } n \text {th datum }\end{array}$ \\
\hline CEEMDAN & $\begin{array}{l}\text { complete ensemble empirical mode decomposition } \\
\text { with adaptive noise }\end{array}$ & $\mathbf{\tau}$ & $\begin{array}{l}\text { delay time } \\
\text { embedding dimension }\end{array}$ \\
\hline EEMD & ensemble empirical mode decomposition & Holt & holt's exponential smoothing \\
\hline EMD & empirical mode decomposition & $S_{t}$ & preliminary predictive value \\
\hline WT & wavelet transfer & $T_{t}$ & local growth value \\
\hline IMF & intrinsic mode function & $L_{t}$ & linear components \\
\hline SVR & support vector regression & $N_{t}$ & non-linear components \\
\hline RBF & radial basic function & $\alpha$ & the smoothing parameter \\
\hline PSO & particle swarm optimization & $\gamma$ & the smoothing parameter \\
\hline FA & firefly algorithm & CTS & chaotic time series \\
\hline $\operatorname{Gen}_{\operatorname{Max}}$ & maximum number of iterations & GRNN & general regression neural network \\
\hline$I_{0}$ & original light intensity. & MLYE & maximum Lyapunov exponent \\
\hline$r_{i j}$ & distance between any two fireflies, $i$ and $j$ & ELM & extreme learning machine \\
\hline $\mathbf{P}$ & number of fireflies & $\mathbf{A N N}$ & artificial neural network \\
\hline SSA & Singular Spectrum Analysis & SVM & support vector machine \\
\hline
\end{tabular}




\section{References}

[1] Xie L, Carvalho PMS, Ferreira L a FM, Juhua Liu, Krogh BH, Popli N, et al. Wind Integration in Power Systems: Operational Challenges and Possible Solutions. Proc IEEE 2011. http://doi:10.1109/JPROC.2010.2070051.

[2] Khalid M, Savkin AV. A method for short-term wind power prediction with multiple observation points. IEEE Trans Power Syst 2012. http://doi:10.1109/TPWRS.2011.2160295.

[3] Ackermann T, Söder L. Wind energy technology and current status: a review. Renew Sustain Energy Rev 2000. http://doi:10.1016/S1364-0321(00)00004-6.

[4] Jung J, Broadwater RP. Current status and future advances for wind speed and power forecasting. Renew Sustain Energy Rev 2014. http://doi:10.1016/i.rser.2013.12.054.

[5] Liu H, Tian Hqi, Li Yfei. Comparison of two new ARIMA-ANN and ARIMAKalman hybrid methods for wind speed prediction. Appl Energy 2012. http://doi:10.1016/j.apenergy.2012.04.001.

[6] Zhao J, Guo ZH, Su ZY, Zhao ZY, Xiao X, Liu F. An improved multi-step forecasting model based on WRF ensembles and creative fuzzy systems for wind speed. Appl Energy 2016. http://doi:10.1016/j.apenergy.2015.10.145.

[7] Yang D, Sharma V, Ye Z, Lim LI, Zhao L, Aryaputera AW. Forecasting of global horizontal irradiance by exponential smoothing, using decompositions. Energy 2015. http://doi:10.1016/j.energy.2014.11.082.

[8] Torres JL, García A, De Blas M, De Francisco A. Forecast of hourly average wind speed with ARMA models in Navarre (Spain). Sol Energy 2005. http://doi:10.1016/j.solener.2004.09.013.

[9] Kavasseri RG, Seetharaman K. Day-ahead wind speed forecasting using f-ARIMA models. Renew Energy 2009. http://doi:10.1016/j.renene.2008.09.006.

[10] Koo J, Han GD, Choi HJ, Shim JH. Wind-speed prediction and analysis based on geological and distance variables using an artificial neural network: A case study in South Korea. Energy 2015. http://doi:10.1016/j.energy.2015.10.026.

[11] Fu T, Wang C. A hybrid wind speed forecasting method and wind energy resource analysis based on a swarm intelligence optimization algorithm and an artificial intelligence model. Sustain 2018;10. http://doi:10.3390/su10113913.

[12]Liu D, Wang J, Wang H. Short-term wind speed forecasting based on spectral clustering and optimised echo state networks. Renew Energy 2015;78:599-608. http://doi:10.1016/j.renene.2015.01.022.

[13] Nikolić V, Motamedi S, Shamshirband S, Petković D, Ch S, Arif M. Extreme learning machine approach for sensorless wind speed estimation. Mechatronics 2015. http://doi:10.1016/j.mechatronics.2015.04.007.

[14] Hu J, Wang J, Ma K. A hybrid technique for short-term wind speed prediction. Energy 2015. http://doi:10.1016/j.energy.2014.12.074.

[15] Wang J, Wang J. Forecasting energy market indices with recurrent neural networks: Case study of crude oil price fluctuations. Energy 2016. http://doi:10.1016/j.energy.2016.02.098.

[16] Wang J, Du P, Niu T, Yang W. A novel hybrid system based on a new proposed algorithm-Multi-Objective Whale Optimization Algorithm for wind speed forecasting. Appl Energy 2017;208:344-60. http://doi:10.1016/j.apenergy.2017.10.031.

[17]Zhang GP. Time series forecasting using a hybrid ARIMA and neural network model. Neurocomputing 2003. http://doi:10.1016/S0925-2312(01)00702-0. 
[18] Li H, Wang J, Lu H, Guo Z. Research and application of a combined model based on variable weight for short term wind speed forecasting. Renew Energy 2018. http://doi:10.1016/j.renene.2017.09.089.

[19] Zhang C, Zhou J, Li C, Fu W, Peng T. A compound structure of ELM based on feature selection and parameter optimization using hybrid backtracking search algorithm for wind speed forecasting. Energy Convers Manag 2017. http://doi:10.1016/i.enconman.2017.04.007.

[20]Zhang W, Qu Z, Zhang K, Mao W, Ma Y, Fan X. A combined model based on CEEMDAN and modified flower pollination algorithm for wind speed forecasting. Energy Convers Manag 2017. http://doi:10.1016/j.enconman.2017.01.022.

[21] Du P, Wang J, Guo Z, Yang W. Research and application of a novel hybrid forecasting system based on multi-objective optimization for wind speed forecasting. $\quad$ Energy $\quad$ Convers 2017. http://doi:10.1016/j.enconman.2017.07.065.

[22] Wang J, Niu T, Lu H, Guo Z, Yang W, Du P. An analysis-forecast system for uncertainty modeling of wind speed: A case study of large-scale wind farms. Appl Energy 2018. http://doi:10.1016/j.apenergy.2017.11.071.

[23] Jiang Y, Huang G. Short-term wind speed prediction: Hybrid of ensemble empirical mode decomposition, feature selection and error correction. Energy Convers Manag 2017;144:340-50. http://doi:10.1016/j.enconman.2017.04.064.

[24] Wang C, Zhang H, Fan W, Ma P. A new chaotic time series hybrid prediction method of wind power based on EEMD-SE and full-parameters continued fraction. Energy 2017. http://doi:10.1016/j.energy.2017.07.112.

[25] Torres ME, Colominas MA, Schlotthauer G, Flandrin P. A complete ensemble empirical mode decomposition with adaptive noise. 2011 IEEE Int Conf Acoust Speech Signal Process 2011. http://doi:10.1109/ICASSP.2011.5947265.

[26]Zhang W, Qu Z, Zhang K, Mao W, Ma Y, Fan X. A combined model based on CEEMDAN and modified flower pollination algorithm for wind speed forecasting. Energy Convers Manag 2017. http://doi:10. 1016/ j. enconman. 2017.01.022.

[27]Ren Y, Suganthan PN, Srikanth N. A comparative study of empirical mode decomposition-based short-term wind speed forecasting methods. IEEE Trans Sustain Energy 2015;6:236-44. http://doi:10.1109/TSTE.2014.2365580.

[28] Guo Z, Chi D, Wu J, Zhang W. A new wind speed forecasting strategy based on the chaotic time series modelling technique and the Apriori algorithm. Energy Convers Manag 2014. http://doi:10.1016/j.enconman.2014.04.028.

[29]Lorenz EN. Deterministic Nonperiodic Flow. J Atmos Sci 1963. http://doi:10.1175/1520-0469(1963)020<0130:DNF>2.0.CO;2.

[30] Tennyson J. In the wake of chaos. Unpredictable order in dynamical systems. Endeavour 1995. http://doi:10.1016/0160-9327(95)90056-X.

[31] Huang N, Shen Z, Long S, Wu M, SHIH H, ZHENG Q, et al. The empirical mode decomposition and the Hilbert spectrum for nonlinear and non-stationary time series analysis. Proc R Soc A Math Phys Eng Sci 1998. http://doi:10.1098/rspa.1998.0193.

[32] Wu Z, Huang NE. Ensemble Empirical Mode Decomposition: A Noise-Assisted Data Analysis Method. Adv Adapt Data Anal 2009. http://doi:10.1142/S1793536909000047.

[33] Wang T, Zhang M, Yu Q, Zhang H. Comparing the applications of EMD and EEMD on time-frequency analysis of seismic signal. J Appl Geophys 2012. http://doi:10.1016/j.jappgeo.2012.05.002.

[34] Jiang P, Li C, Li R, Yang H. An innovative hybrid air pollution early-warning 
system based on pollutants forecasting and Extenics evaluation. Knowledge-Based Syst 2018. http://doi:10.1016/i.knosys.2018.10.036.

[35] Takens F. Dynamical systems and turbulence lecture notes in mathematics. Berlin:Springer; 1981. 366e81.

[36] Kim HS, Eykholt R, Salas JD. Nonlinear dynamics, delay times, and embedding windows. Phys D Nonlinear Phenom 1999. http://doi:10.1016/S01672789(98)00240-1.

[37] Yang X-S. Nature-Inspired Metaheuristic Algorithms. Nature-Inspired Metaheuristic Algorithms Second Edition 2010. http://doi:10.1016/B978-0-12416743-8.00005-1.

[38] Holt CC. Forecasting seasonals and trends by exponentially weighted moving averages. Int J Forecast 2004. http://doi:10.1016/j.ijforecast.2003.09.015.

[39] Vapnik VN. The nature of statistical learning theory. IEEE Trans Neural Networks 1995;8:1564-1564. http://doi:10.1109/TNN.1997.641482.

[40] Chen K-Y. Combining linear and nonlinear model in forecasting tourism demand. Expert Syst Appl 2011. http://doi:10.1016/j.eswa.2011.02.049.

[41] Wang J, Du P, Lu H, Yang W, Niu T. An improved grey model optimized by multiobjective ant lion optimization algorithm for annual electricity consumption forecasting. Appl Soft Comput J 2018;72:321-37. http://doi:10.1016/j.asoc.2018.07.022.

[42] Jiang P, Yang H, Heng J. A hybrid forecasting system based on fuzzy time series and multi-objective optimization for wind speed forecasting. Appl Energy 2019;235:786-801. http://doi:10.1016/j.apenergy.2018.11.012.

[43]Zhao X, Wang C, Su J, Wang J. Research and Application Based on the Swarm Intelligence Algorithm and Artificial Intelligence for Wind Farm Decision System. Renew Energy 2018. http://doi:10.1016/j.renene.2018.11.061.

[44] Wang Y, Wu L. On practical challenges of decomposition-based hybrid forecasting algorithms for wind speed and solar irradiation. Energy 2016;112:208-20. http://doi:10.1016/j.energy.2016.06.075.

[45] Sun S, Qiao H, Wei Y, Wang S. A new dynamic integrated approach for wind speed forecasting. Appl Energy 2017;197:151-62. http://doi:10.1016/j.apenergy.2017.04.008.

[46] Wang Y, Wang J, Wei X. A hybrid wind speed forecasting model based on phase space reconstruction theory and Markov model: A case study of wind farms in northwest China. Energy 2015. http://doi:10.1016/j.energy.2015.08.039.

[47] Yang X. Firefly Algorithm. 2010. http://doi:10.1016/B978-0-12-416743-8.000051.

[48]Zhang W, Qu Z, Zhang K, Mao W, Ma Y, Fan X. A combined model based on CEEMDAN and modified flower pollination algorithm for wind speed forecasting. Energy Convers Manag 2017. http://doi:10.1016/j.enconman.2017.01.022. 